\title{
Doctor, Teacher, and Stethoscope: Neural Representation of Different Types of Semantic Relations
}

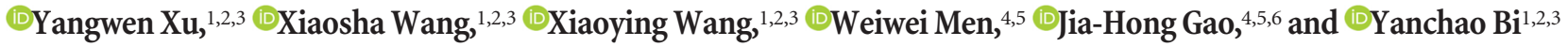 \\ ${ }^{1}$ National Key Laboratory of Cognitive Neuroscience and Learning, Beijing Normal University, Beijing, China, 100875, ${ }^{2}$ IDG/McGovern Institute for Brain \\ Research, Beijing Normal University, Beijing, China, 100875, ${ }^{3}$ Beijing Key Laboratory of Brain Imaging and Connectomics, Beijing Normal University, \\ Beijing, China, 100875, ${ }^{4}$ Center for MRI Research, Academy for Advanced Interdisciplinary Studies, Peking University, Beijing, China, 100871, ${ }^{5}$ Beijing City \\ Key Laboratory for Medical Physics and Engineering, Institute of Heavy Ion Physics, School of Physics, Peking University, Beijing, China, 100871, and ${ }^{6}$ IDG/ \\ McGovern Institute for Brain Research, Peking University, Beijing, China, 100871
}

Concepts can be related in many ways. They can belong to the same taxonomic category (e.g., "doctor" and "teacher," both in the category of people) or be associated with the same event context (e.g., "doctor" and "stethoscope," both associated with medical scenarios). How are these two major types of semantic relations coded in the brain? We constructed stimuli from three taxonomic categories (people, manmade objects, and locations) and three thematic categories (school, medicine, and sports) and investigated the neural representations of these two dimensions using representational similarity analyses in human participants (10 men and nine women). In specific regions of interest, the left anterior temporal lobe (ATL) and the left temporoparietal junction (TPJ), we found that, whereas both areas had significant effects of taxonomic information, the taxonomic relations had stronger effects in the ATL than in the TPJ ("doctor" and "teacher" closer in ATL neural activity), with the reverse being true for thematic relations ("doctor" and "stethoscope" closer in TPJ neural activity). A whole-brain searchlight analysis revealed that widely distributed regions, mainly in the left hemisphere, represented the taxonomic dimension. Interestingly, the significant effects of the thematic relations were only observed after the taxonomic differences were controlled for in the left TPJ, the right superior lateral occipital cortex, and other frontal, temporal, and parietal regions. In summary, taxonomic grouping is a primary organizational dimension across distributed brain regions, with thematic grouping further embedded within such taxonomic structures.

Key words: category; RSA; semantic relation; semantics; taxonomic; thematic

Significance Statement

How are concepts organized in the brain? It is well established that concepts belonging to the same taxonomic categories (e.g., "doctor" and "teacher") share neural representations in specific brain regions. How concepts are associated in other manners (e.g., "doctor" and "stethoscope," which are thematically related) remains poorly understood. We used representational similarity analyses to unravel the neural representations of these different types of semantic relations by testing the same set of words that could be differently grouped by taxonomic categories or by thematic categories. We found that widely distributed brain areas primarily represented taxonomic categories, with the thematic categories further embedded within the taxonomic structure.

\section{Introduction}

The relations among concepts are critical elements of the semantic space. Numerous neuropsychological and neuroimaging studies

Received Sept. 6, 2017; revised Feb. 8, 2018; accepted Feb. 13, 2018.

Author contributions: Y.X., Xiaosha Wang, Xiaoying Wang, and Y.B. designed research; Y.X., W.M., and J.-H.G. performed research; Y.X. and Y.B. analyzed data; Y.X., Xiaosha Wang, Xiaoying Wang, and Y.B. wrote the paper.

This work was supported by the National Key Basic Research Program of China (Grants 2013 CB837300 and 2014 (B846100 to Y.B.), the National Natural Science Foundation of China (Grant 31671128 to Y.B., Grant 31700943 to Xiaosha Wang, Grant 31500882 to Xiaoying Wang, and Grants $81430037,81727808,81790650$ and 81790651 to J.-H.G.), the China Postdoctoral Science Foundation (Grant 2017 M610791 to X.S.W), the National Program for Special Support of Top-notch Young Professionals (Y.B.), the Fundamental Research Funds for the Central Universities (Grant 2017XTCX04 to Y.B.), and the Interdisciplinary Research Funds of Beijing Normal University and Beijing have shown that semantically related concepts are represented by shared neural substrates (Rudrauf et al., 2008; Binder et al., 2009; Fairhall and Caramazza, 2013a) or induce similar neural activation patterns (Shinkareva et al., 2011; Devereux et al., 2013;

Advanced Innovation Center for Future Education (Grant BJAICFE2016IR-003 to Y.B.). We thank Wei Wu, Xiaonan Li, Yan Chen, and Ye Li for assistance with data collection.

The authors declare no competing financial interests.

Correspondence should be addressed to Yanchao Bi, National Key Laboratory of Cognitive Neuroscience and Learning, IDG/McGovern Institute for Brain Research, and Beijing Key Laboratory of Brain Imaging and Connectomics, Beijing Normal University, Beijing 100875, China. E-mail: ybi@bnu.edu.cn.

DOI:10.1523/JNEUROSCI.2562-17.2018

Copyright $\odot 2018$ the authors $\quad 0270-6474 / 18 / 383303-15 \$ 15.00 / 0$ 
Fairhall and Caramazza, 2013b; Simanova et al., 2014). However, most studies have only focused on taxonomic relations, in which concepts sharing similar features belong to the same taxonomic categories (Rogers and McClelland, 2004; Binder et al., 2016). For example, "doctor" and "teacher" are both kinds of people and share many more features relative to items from other taxonomic categories such as tools. "Doctor" is also semantically related to "stethoscope," not based on feature similarity, but rather because they often cooccur in the same scenario or event. This relation is considered to be thematic (Estes et al., 2011). These two types of semantic relations constitute two major organizational dimensions of semantic contents; unraveling the corresponding neural substrates is necessary to elucidate the neural mechanisms of semantic representation.

Previous neuroimaging and neuropsychological studies have failed to reach a consensus on the neural basis of taxonomic and thematic relations (for review, see Mirman et al., 2017). Several studies have reported neuroanatomical dissociation between taxonomic and thematic relations, with the anterior temporal lobe (ATL) being sensitive to taxonomic representation and the temporoparietal junction (TPJ) being the reverse (Schwartz et al., 2011; Mirman and Graziano, 2012; Geng and Schnur, 2016). Others have reported different patterns. Some studies found effects in only one of these two regions (Kalénine et al., 2009; de Zubicaray et al., 2013; Lewis et al., 2015); some found many other regions that were associated with either type of semantic relations but did not show a clear convergence (Kotz et al., 2002; Sachs et al., 2008a,b, 2011; Kalénine et al., 2009; Kuchinke et al., 2009; Sass et al., 2009; Anderson et al., 2014; de Zubicaray et al., 2014; Kalénine and Buxbaum, 2016); and some failed to detect any neural dissociations between these two types of relations (Jackson et al., 2015). Several factors have been considered to give rise to such discrepancies, including the confounding factors of behavioral difference (Jackson et al., 2015), the semantic processing depth (Geng and Schnur, 2016), and the inconsistent definition of thematic relations (Mirman et al., 2017). Critically, these studies have predominantly used univariate contrasts between the activation strengths in different conditions, which may mask effects for which the two kinds of relations are represented through different activation patterns rather than overall activity strength. The only multivariate study (Anderson et al., 2014) focused on the whole-brain pattern without specific contrasts between the ATL and the TPJ.

Addressing these issues, we used the representational similarity analysis (RSA) (Kriegeskorte et al., 2008; Mur et al., 2009) to unravel brain regions where concepts are related along taxonomic or thematic dimensions (Fig. 1), with attention paid to behavioral differences, semantic tasks, and definitions of thematic relations. Nine sets of words were constructed across three taxonomic categories (people, manmade objects, and locations) and three thematic categories (school, medicine, and sports). Participants were instructed to explicitly access semantic information along either taxonomic or thematic dimensions in different runs. The trial-by-trial reaction time (RT) differences were controlled for. Theoretical representation dissimilarity matrices (RDMs) were constructed based on either of these two dimensions (e.g., the taxonomic RDM: doctor-teacher, 0, doctor-stethoscope, 1; the thematic RDM: doctor-teacher, 1, doctor-stethoscope, 0 ). The relations between these theoretical RDMs and the neural RDMs were examined in ROIs (the left ATL and TPJ); in a larger scale of potential neural encoding, i.e., modules of the semantic network (Xu et al., 2016); and in a whole-brain searchlight fashion. We specifically examined whether the two semantic dimensions had any relatively unique effects.

\section{Materials and Methods}

Participants. Twenty healthy native Chinese speakers (10 males; aged 18-27 years) participated in this study. All participants were right handed (measured by a Chinese adaptation of Oldfield, 1971) and had normal or corrected-to-normal vision. All participants provided written informed consent. This study was approved by the Human Subject Review Committee at Peking University. One participant (female) was excluded from the analyses due to a technical dysfunction.

Stimuli. The stimuli set contained 45 Chinese words that belonged to nine conditions: three taxonomic categories (people, manmade objects, and locations) $\times$ three thematic categories (school, medicine, and sports). Each condition had five words, including three bisyllabic (two characters) words and two trisyllabic (three characters) words, thus matching on number of syllables and characters across all conditions. Words across three taxonomic $\times$ three thematic categories were well matched on their visual complexity (stroke numbers among taxonomic categories: $F_{(2,36)}=0.09, p=0.914$; among thematic categories: $F_{(2,36)}=0.92, p=0.409$; interaction: $F_{(4,36)}=0.29, p=0.881$ or logographeme numbers among taxonomic categories: $F_{(2,36)}=0.82, p=$ 0.449 ; among thematic categories: $F_{(2,36)}=0.25, p=0.782$; interaction: $\left.F_{(4,36)}=0.61, p=0.658\right)$. To further ensure that any semantic condition effects were not attributable to potential confounding variables such as visual shape or word frequency, we performed validation analyses (see Results section) including the following matrices as covariates using Spearman's rank partial correlation: (1) the low-level visual dissimilarity matrix (Kriegeskorte et al., 2008; Devereux et al., 2013), in which each cell represented the mean pairwised Jaccard distance between the binary silhouette images of words across conditions (i.e., mean of 25 word pairs in each pair of conditions), and (2) the word frequency dissimilarity matrix, in which each cell contained the mean pairwise difference between the word frequency counts (Sun et al., 1997) across conditions. Finally, words in the sports condition, such as "award platform" and "audience," tended to be associated with boarder thematic situations, so we presented all words with pictures depicting the intended meanings in a familiarization phase and performed further validation analyses excluding the sport condition (see Results section). Each word was presented visually in black "Song" bold, 36-point sized font at the center of a gray background. The viewing distance was $1.1 \mathrm{~m}$.

Procedures. Before scanning, we had a warm-up session that presented each word with a picture of its intended meaning to familiarize the participants with the stimuli and to resolve potential ambiguities (see above). There were 10 runs during the scanning. Each run lasted 260 s. A $10 \mathrm{~s}$ blank screen was presented at the start and end of each run. All stimuli were presented once during each run. We first determined the sequence of the nine conditions in each run using the optseq 2 optimization algorithm (Dale, 1999). The presenting orders of the five words within each condition were further randomized. The run orders were randomized across participants. Each trial started with a centrally presented fixation cross on a gray background for $500 \mathrm{~ms}$, followed by a word stimulus for $500 \mathrm{~ms}$, and then a blank screen with varying lengths between 3 and $13 \mathrm{~s}$, which were also defined using the optseq 2 optimization algorithm. The participants were instructed to make semantic judgments (see below) in the subsequent $3500 \mathrm{~ms}$ after the onset of each stimulus. Both accuracy and RT were recorded. These procedures were implemented using E-prime 2.

Tasks. To ensure that the factor of the tasks did not bias the results, the participants were instructed to assess the meaning of these stimuli along both dimensions. In half of runs, a taxonomic judgment task was performed. The participants pressed the buttons with their right middle finger, right index finger, and left index finger when the stimuli belonged to the taxonomic categories of man made objects, people, and locations, respectively. In the other half of runs, the participants pressed the buttons with their right middle finger, right index finger, and left index finger when the stimuli belonged to the thematic categories of medicine, school, and sports, respectively. The sequence of the taxonomic and thematic runs was randomized across the participants. 
A

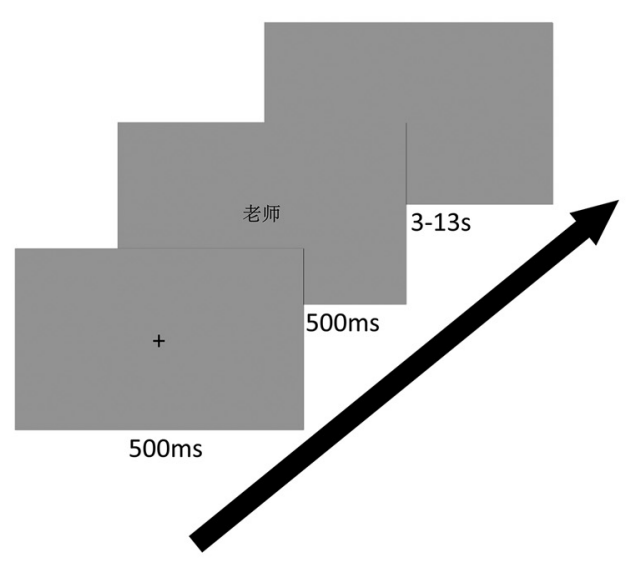

B

\begin{tabular}{|c|c|c|c|}
\hline & School & Medicine & Sports \\
\hline \multirow{2}{*}{ People } & $\begin{array}{c}\text { Teacher } \\
\text { Student } \\
\text { Professor } \\
\text { Class Advisor }\end{array}$ & $\begin{array}{c}\text { Doctor } \\
\text { Patient }\end{array}$ & $\begin{array}{c}\text { Referee } \\
\text { Coach }\end{array}$ \\
& Subject Representative & Anesthetist & Audience \\
Midwife & Athlete \\
Cheerleaders
\end{tabular}

C

Taxonomic RDM

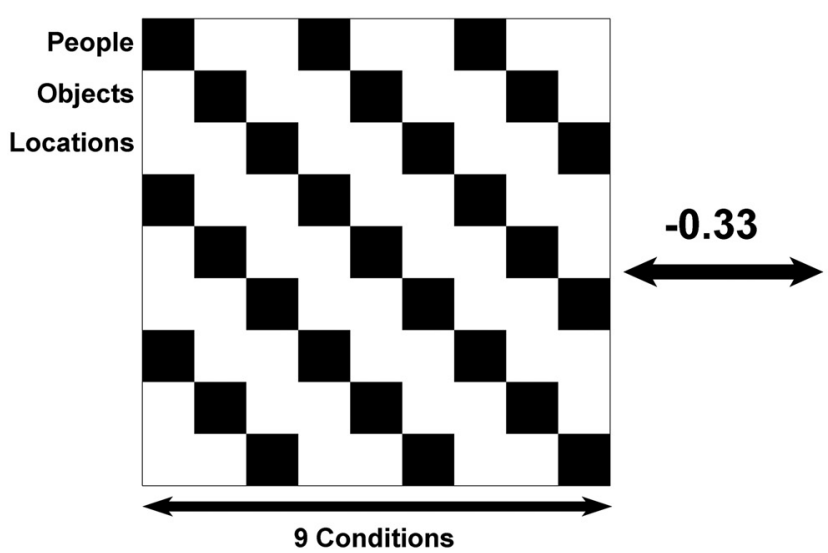

Thematic RDM

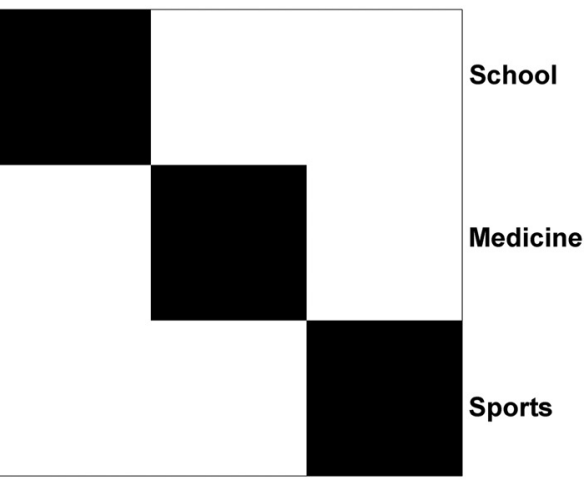

Similar
Dissimilar

Figure 1. Experimental design. A, Procedures in a trial. Each trial started with a fixation cross on a gray background for $500 \mathrm{~ms}$, followed by the stimulus for $500 \mathrm{~ms}$ and then a blank screen lasting between 3 and 13 s. This interval was defined using optseq2 (Dale, 1999). B, Stimuli. Forty-five words were used and were organized into nine conditions (three taxonomic $\times$ three thematic categories). C, Hypothetical RDM. The taxonomic RDM was grouped by taxonomic relations and the thematic RDM was grouped by thematic relations. They were negatively correlated.

Image acquisition. Imaging data were acquired using a MAGNETOM Prisma 3T MR scanner (Siemens) with 20-channel head-neck coil at the Centre for MRI Research, Peking University. High-resolution functional images were acquired using the simultaneous multislices echoplanar imaging sequence, the scanning plane is parallel to the straight gyrus, the phase encoding direction from posterior to anterior, repetition time $(\mathrm{TR})=2000 \mathrm{~ms}$, echo time $(\mathrm{TE})=30 \mathrm{~ms}$, flip angle $(\mathrm{FA})=90^{\circ}$, field of view $(\mathrm{FOV})=224 \mathrm{~mm} \times 224 \mathrm{~mm}$, matrix size $=112 \times 112,64$ axial slices, slices thickness $(\mathrm{ST})=2 \mathrm{~mm}$, gap $=0.2 \mathrm{~mm}$, voxel size $=2 \times 2 \times$ $(2+0.2) \mathrm{mm}$, multiband factor $=2$. High-resolution 3D T1-weighted images were acquired using the magnetization-prepared rapid gradientecho sequence, sagittal plane, $\mathrm{TR}=2530 \mathrm{~ms}, \mathrm{TE}=2.98 \mathrm{~ms}$, inversion time $=1100 \mathrm{~ms}, \mathrm{FA}=7^{\circ}, \mathrm{FOV}=224 \mathrm{~mm} \times 256 \mathrm{~mm}$, matrix size $=$ $224 \times 256$, interpolated to $448 \times 512,192$ continuous sagittal slices, $\mathrm{ST}=1 \mathrm{~mm}$, voxel size $=0.5 \times 0.5 \times 1 \mathrm{~mm}$.

Image preprocessing. The images were preprocessed using SPM12 (Wellcome Trust Center for Neuroimaging, http://www.fil.ion.ucl.ac. $\mathrm{uk} / \mathrm{spm} / \mathrm{software} / \mathrm{spm} 12 /$ ). Functional images were processed using procedures including first five volume exclusion, slice timing correction, and realignment to the individual's first image of their first run using six rigid body-transforming parameters. These resulting unsmoothed and unnormalized images were entered into the general linear model (GLM) at the individual level to maintain the original informative pattern across the voxels (Kriegeskorte et al., 2006). The GLM contained a regressor for each of these nine conditions, along with six head motion regressors and a constant regressor for each run. To investigate the effects of the type of semantic task on the neural representational patterns, we also built another GLM in which the same stimuli conditions under different tasks (the taxonomic judgment task and the thematic judgment task) were treated as different repressors. To control for potential confounding effects of the RT, we used the duration modulation method by convolving each trial with a boxcar equal to the length of the trial's RT for each participant (Grinband et al., 2008). A high-pass filter cutoff was set as $128 \mathrm{~s}$. To ensure maximal coverage of the ATL, a lower threshold (10\% of the mean global signal) was adopted as the implicit mask (Devereux et al., 2013). To suppress the contribution of noisy voxels with high $\beta$ estimates due to high noise (Misaki et al., 2010), the $t$-value image of each condition was calculated to capture the activation patterns. The structure images were coregistered to the mean functional images and segmented into different tissues. The resulting gray matter probabilistic image of each participant was resliced into the same resolution as that of the functional images and thresholded at one-third to generate a binary mask for the searchlight-based RSA. The forward and inverse deformation fields of each participant's native space to the Montreal Neurological Institute (MNI) space were also obtained in this step.

Multivoxel pattern analyses (MVPA). For MVPA, RSA and multidimensional scaling (MDS) were implemented. For any brain region at the MNI space, the images were first transformed to each participant's native space via inverse deformation fields and resliced to the same resolution as that of the functional images. 
RSA. The RSA was used to identify the representational content that emerged from the multivariate activation patterns across the voxels in a given brain area (Kriegeskorte et al., 2008; Mur et al., 2009). The representation of a brain area was characterized by the RDM, which is a symmetric matrix indexed by these nine conditions horizontally and vertically in the same order. Each element in this matrix measured the dissimilarity between the activation patterns in the two conditions across all voxels in that brain area. The Spearman's rank correlation distance ( 1 - Spearman's $r$ ) was used to measure this dissimilarity. Then, the brain RDM was compared with multiple model RDMs by calculating the Spearman's rank correlation across the elements within the lower triangle (not including the diagonal) or calculating the Spearman's rank partial correlation to control for certain variables. The resulting correlation coefficients were Fisher transformed and statistically inferred across participants. We mostly focused on two hypothetical model RDMs (Fig. 1), the taxonomic RDM grouped by the taxonomic categories (e.g., teacherdoctor, 0 ; teacher-chalk, 1) and the thematic RDM grouped by the thematic categories (e.g., teacher-doctor, 1; teacher-chalk, 0). These two model RDMs were negatively correlated (Spearman's $r=-0.33$ ). The RSA results will reflect whether the neural patterns associated with the taxonomic RDM or the thematic RDM.

MDS. An MDS analysis was implemented to provide a visualization of the representational patterns of a particular brain region. For any given brain region, an MDS analysis was applied on the mean neural RDM across participants using the PROXSCAL procedure (Busing et al., 1997) in SPSS Statistics 22. The PROXSCAL performs multidimensional scaling of proximity data to find a least-square representation of the objects in a low-dimensional space by minimizing the normalized raw stress. Ten thousand random starts were configured and the configuration with the lowest normalized raw stress was used as the initial configuration.

ROI definition. We focused on two anatomically defined ROIs: the left ATL and the left TPJ. The left ATL was defined as the union set of the following six subregions according to the Harvard-Oxford Atlas (probability $>0.2)$ : the temporal pole (TP), the anterior superior temporal gyrus (aSTG), the anterior middle temporal gyrus (aMTG), the anterior inferior temporal gyrus (aITG), the anterior temporal fusiform cortex (aTFC), and the anterior parahippocampal gyrus (aPHG) (see Figs. 2A, $3 A$ ). These areas cover the regions of Brodmann area (BA) 38 and the anterior portions of BA 20 and BA 21, in which taxonomic errors are localized (Schwartz et al., 2011). The left TPJ was defined as the union of the posterior supramarginal gyrus (pSMG) and the angular gyrus (AG) in the Harvard-Oxford atlas (probability > 0.2) (see Figs. $2 A, 3 D$ ). These areas cover the regions of BA 39 and the adjacent supramarginal gyrus, in which thematic errors are localized (Schwartz et al., 2011). To determine whether different subregions in the left ATL and the left TPJ varied, we also investigated the representational patterns in each subregion of the two regions separately. Because the ATL is adjacent to the air-bone interface that leads to inhomogeneities in the magnetic field, we computed the temporal signal-to-noise ratio (tSNR) in each subregion of the ATL for each participant using the motion-corrected unsmoothed images in the native space. The tSNR was calculated by dividing the mean of the time series across the whole run by its SD (Murphy et al., 2007). The mean tSNR over all voxels within a subregion and over all the runs represented the $\mathrm{tSNR}$ of that subregion.

Whole-brain searchlight. A whole-brain searchlight-based RSA was implemented under the framework of Kriegeskorte et al. (2006) to identify brain areas that locally represent taxonomic and thematic relations and their unique information. A whole-brain searchlight was implemented within the individual gray matter mask generated in the preprocessing stage. For each voxel, a 6-mm-radius sphere was built (including 113 voxels) and the activity patterns of these voxels across different conditions were extracted to build the neural RDM. These neural RDMs were correlated with model RDMs and the Fisher-transformed Spearman's correlation coefficient was returned to this voxel. The resulting similarity maps of each participant were normalized to the MNI space using the forward deformation field and were spatially smoothed using a $6 \mathrm{~mm}$ full-width at half-maximum Gaussian kernel. A group-level randomeffect analysis was then implemented across these maps using the permutation-based nonparametric method (Nichols and Holmes, 2002) with SnPM 13 (http://warwick.ac.uk/snpm). No variance smoothing was used and 10,000 permutations were performed. A conventional clusterextent-based inference threshold (voxel level at $p<0.001$; cluster-extent FWE $p<0.05$ ) was adopted and we stated explicitly when more stringent or moderate thresholds were applied. We also performed searchlight analyses using spheres with radii of 4,8 , and $10 \mathrm{~mm}$. The results using the different-sized spheres were very similar to each other with the tendency that the effects became stronger as the radius increased.

Given that participants were instructed to press different buttons according to taxonomic or thematic categories in the taxonomic and thematic judgment tasks, respectively, the effect of button press inevitably confounded the taxonomic and the thematic effects in the corresponding task. To exclude the confounding factors of button press, we performed the following analyses. First, we localized effects that are associated with button press across tasks. The model RDM of button press was defined as the rank variables according to whether the participants pressed the button using the same hand and finger (same hand, same finger: 0; same hand, different finger: 1; different hand, different finger: 2). Therefore, the taxonomic task and the thematic task had different button press RDMs. They were not positive correlated (Spearman's $r=-0.22$ ) and approximately corresponded to the taxonomic RDM in the taxonomic task and the thematic RDM in the thematic task. We correlated these two button press RDMs with the neural representational pattern in their corresponding tasks. The button press effect should be the common areas of these two correlations. Any clusters with semantic RSA effects overlapping with these areas should be excluded. Second, we further validated the main results with an additional analysis in a cross-task-andcondition fashion. That is, we performed the RSA using the taxonomic RDM correlated with the neural activity pattern in the thematic tasks and using the thematic RDM correlated with the neural activity pattern in the taxonomic tasks. Because the button press RDM of one semantic task was not positively correlated with the semantic RDM in the other type (Spearman's $r=-0.27$ ), the effects of button press would not confound the semantic relation effects.

Semantic subnetwork definition. For regions that are consistently activated during semantic tasks (Binder et al., 2009), based on the topological structures of their connections, Xu et al. (2016) identified three intrinsic semantic subnetworks, including a "semantic-DMN module" and a "semantic-perisylvian network (PSN) module," which were hypothesized to support semantic representation. Masks of these two modules were adopted based on the voxelwise results under a connectivity density of 0.4 (see Fig. 5A). The semantic-DMN subnetwork primarily includes the bilateral retrosplenial cortices/posterior cingulate cortices/ precuneus, bilateral medial prefrontal cortices, bilateral posterior angular gyrus extending to the transverse occipital sulcus, the left superior frontal gyrus, and the middle part of the left fusiform gyrus/parahippocampal cortex. The semantic-PSN subnetwork primarily includes the left ventral frontal cortices, the entire length of the left middle temporal cortices, and the left TPJ.

Brain visualization. The brain results were mapped onto the inflated surface of the PALS-B12 atlas (Van Essen, 2005) using Caret (http:// brainvis.wustl.edu/wiki/index.php/Caret:Download). We used the average fiducial map to display the whole-brain searchlight results.

\section{Results}

\section{Behavioral results}

The means and SDs of RT and accuracy in each condition are presented in Table 1 . We performed two tasks $\times$ three taxonomic $\times$ three thematic categories repeated-measures ANOVA. Regarding accuracy, analyses using the arcsine transformed accuracy data revealed no significant effects of any main effects or three-way interactions $(p>0.05)$. Regarding the RT, there was no significant effect of tasks or three-way interactions $(p>0.1)$, but significant main effects of taxonomic categories $\left(F_{(2,27)}=\right.$ $10.42, p=0.001$, Greenhouse-Geisser corrected for the degrees of freedom $)$ and of thematic categories $\left(F_{(2,36)}=3.41, p=0.044\right)$. 
Table 1. Accuracy (ACC) and RT data (mean \pm SD)

\begin{tabular}{|c|c|c|c|c|c|}
\hline \multirow[b]{2}{*}{$\begin{array}{l}\text { Taxonomic } \\
\text { category }\end{array}$} & \multirow[b]{2}{*}{$\begin{array}{l}\text { Thematic } \\
\text { category }\end{array}$} & \multicolumn{2}{|c|}{$\begin{array}{l}\text { Taxonomic } \\
\text { judgment task }\end{array}$} & \multicolumn{2}{|c|}{$\begin{array}{l}\text { Thematic } \\
\text { judgment task }\end{array}$} \\
\hline & & $\mathrm{ACC}(\%)$ & RT (ms) & ACC (\%) & $\mathrm{RT}$ (ms) \\
\hline \multirow[t]{3}{*}{ People } & School & $98 \pm 3$ & $1460 \pm 420$ & $95 \pm 7$ & $1434 \pm 447$ \\
\hline & Medicine & $95 \pm 5$ & $1480 \pm 408$ & $95 \pm 6$ & $1499 \pm 432$ \\
\hline & Sports & $97 \pm 4$ & $1555 \pm 433$ & $95 \pm 5$ & $1580 \pm 419$ \\
\hline \multirow[t]{3}{*}{ Manmade objects } & School & $96 \pm 5$ & $1506 \pm 376$ & $94 \pm 5$ & $1493 \pm 442$ \\
\hline & Medicine & $99 \pm 3$ & $1424 \pm 429$ & $98 \pm 3$ & $1420 \pm 442$ \\
\hline & Sports & $98 \pm 3$ & $1480 \pm 405$ & $96 \pm 6$ & $1523 \pm 410$ \\
\hline \multirow[t]{3}{*}{ Locations } & School & $92 \pm 8$ & $1573 \pm 407$ & $96 \pm 4$ & $1550 \pm 463$ \\
\hline & Medicine & $97 \pm 3$ & $1582 \pm 454$ & $96 \pm 5$ & $1516 \pm 417$ \\
\hline & Sports & $94 \pm 11$ & $1584 \pm 391$ & $97 \pm 5$ & $1461 \pm 429$ \\
\hline
\end{tabular}

To exclude the potential confounding effect of the trial-bytrial RT difference (Yarkoni et al., 2009; Todd et al., 2013), we used the duration modulation method (Grinband et al., 2008) in the subsequent brain analyses (see Material and Methods). To exclude the potential confounding effect of the accuracy differences across conditions on RSA, we implemented a validation analysis using the Spearman partial correlation to control for the accuracy RDMs of individual participants, in which each cell represented the pairwise accuracy differences between conditions (see "Validation analyses" section below).

\section{Taxonomic and thematic representation in the left ATL and left TPJ}

We first investigated the representational patterns in two anatomically defined ROIs, the left ATL and left TPJ (Fig. 2A).

\section{Left ATL is primarily organized by the taxonomic dimension and} modulated by the thematic dimension

As shown in Figure $2 B$, the RSA revealed that the neural response pattern in the left ATL was significantly correlated with the taxonomic RDM (mean Fisher-transformed Spearman's $r=0.24$; $t_{(18)}=4.80, p<0.001$ ) and not with the thematic RDM (mean $\left.r=0.01 ; t_{(18)}=0.24, p=0.817\right)$. The strength of its correlation with the taxonomic RDM was significantly greater than that with the thematic RDM (paired $t_{(18)}=2.76, p=0.013$ ). After controlling for the thematic difference using Spearman's rank partial correlation, the taxonomic effect remained significant (partial correlation, mean $r=0.25 ; t_{(18)}=5.91, p<0.001$ ); after controlling for the taxonomic difference, the thematic effect became significant (partial correlation, mean $r=0.09, t_{(18)}=2.87, p=$ $0.010)$. The MDS results also illustrated that the representational pattern in the left ATL was largely grouped by the taxonomic dimension (Fig. 2E, left; normalized raw stress $=0.092$, stress- $\mathrm{I}=$ 0.303 , stress-II $=0.942$, S-stress $=0.246$ ).

Analyses within different subregions of the left ATL showed that all subregions represented taxonomic information (mean $r$ : 0.11-0.21; one-sample $t$ test against zero, Bonferroni-corrected $p<0.03$ ), but not thematic information (mean $r$ : -0.01 to 0.01 ; one-sample $t$ test against zero, uncorrected $p>0.41$ ) (Fig. $3 C$ ). A six subregions $X$ two types of semantic information (taxonomic and thematic information) repeated-measures ANOVA revealed a significant effect of semantic information $\left(F_{(1,18)}=9.17, p=\right.$ $0.007)$, but not the main effect of subregions $\left(F_{(5,90)}=1.938, p=\right.$ $0.096)$ or the interaction between subregions and semantic information $\left(F_{(5,90)}=0.48, p=0.790\right)$. After controlling for the thematic difference, all subregions in the left ATL continued to have significant effects of taxonomic information (partial correlation, mean $r$ : 0.12-0.23; one-sample $t$ test, Bonferroni corrected $p<$
0.02). After controlling for the taxonomic difference, some subregions in the left ATL, i.e., the aSTG, the aMTG, and the aPHG, showed trends of thematic information effect (partial correlation, mean $r$ : 0.07 in aSTG, 0.07 in aMTG, and 0.10 in aPHG; one-sample $t$ test, uncorrected $p<0.04$ ) that did not survive the Bonferroni correction. We also calculated the tSNR in each subregion in the left ATL (Fig. $3 B$ ). The semantic information across subregions here did not seem to be fully associated with tSNR; the correlation between the tSNR and the amount of semantic information (sum of the RSA value of both taxonomic and thematic effects) across regions were not significant (Spearman's $r=0.37$, $p=0.47$ ); subregions such as the aPHG had low tSNR yet trends of greater amount of semantic information.

Left TPJ represents taxonomic and thematic information equally As shown in Figure $2 C$, the RSA results showed that the neural response pattern in the left TPJ was significantly correlated with both the taxonomic RDM (mean $r=0.15 ; t_{(18)}=3.60, p=0.002$ ) and the thematic RDM (mean $r=0.09 ; t_{(18)}=2.30, p=0.034$ ) without significant differences between these two effects (paired $t_{(18)}=0.82, p=0.422$ ). After controlling for the thematic difference, the taxonomic effects remained significant (partial correlation, mean $r=0.20 ; t_{(18)}=5.53, p<0.001$ ), and, after controlling for the taxonomic difference, the thematic effect was also significant (partial correlation, mean $r=0.15 ; t_{(18)}=4.75$, $p<0.001$ ). The MDS results also illustrated that the representational pattern in the left TPJ appeared to reflect both taxonomic and thematic dimensions (Fig. $2 E$, right; normalized raw stress = 0.089 , stress-I $=0.299$, stress-II $=0.928$, S-stress $=0.238$ ).

Analyses in different subregions within TPJ also showed that the pattern observed using the whole TPJ was rather homogeneous, with no significant differences across the two subregions (Fig. 3E). Both the left pSMG and the left AG activity patterns contained taxonomic (mean $r=0.14$ in pSMG, mean $r=0.17$ in AG; one-sample $t$ test, $p \leq 0.002$ ) and thematic information (mean $r=0.09$ in pSMG, mean $r=0.06$ in AG; one-sample $t$ test, $p<0.057$ ). The two subregions (the left pSMG and the left AG) $\times$ two types of semantic information (taxonomic and thematic information) repeated-measures ANOVA did not reveal any significant effects (main effect of subregions: $F_{(1,18)}=1.48, p=0.240$; main effect of types of semantic information: $F_{(1,18)}=0.08, p=$ 0.781; interaction between regions and types of semantic information: $\left.F_{(1,18)}=1.77, p=0.200\right)$. After controlling for the thematic difference, both subregions showed effects of taxonomic information (partial correlation, mean $r=0.18$ in pSMG, mean $r=0.21$ in AG; one-sample $t$ test, $p<0.001$ ). After controlling for the taxonomic difference, both subregions also showed effects of thematic information (partial correlation, mean $r=0.15$ in pSMG, mean $r=0.13$ in AG; one-sample $t$ test, $p \leq 0.001$ ).

\section{Comparisons between the left ATL and TPJ}

Figure $2 D$ displays the results of the direct comparisons between the two ROIs. A two regions (left ATL and left TPJ) $\times$ two types of semantic dimension (taxonomic and thematic information) repeated-measures ANOVA revealed a significant interaction $\left(F_{(1,18)}=7.87, p=0.012\right)$. The main effect of types of semantic dimension was marginally significant $\left(F_{(1,18)}=3.79, p=0.067\right)$ and the main effect of regions was not $\left(F_{(1,18)}=0.004, p=0.953\right)$. The post hoc analyses revealed that the left ATL carried more taxonomic information than the left TPJ (paired $t_{(18)}=2.61, p=$ 0.018 ), whereas the left TPJ carried more thematic information than the left ATL (paired $t_{(18)}=2.48, p=0.023$ ). Although the tSNR of the ATL was significantly lower than that of the TPJ 
A

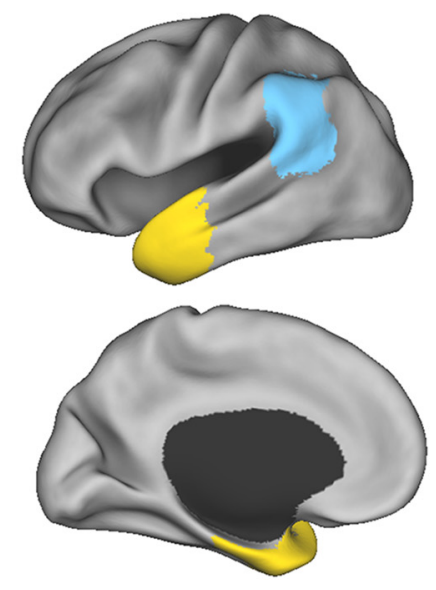

C

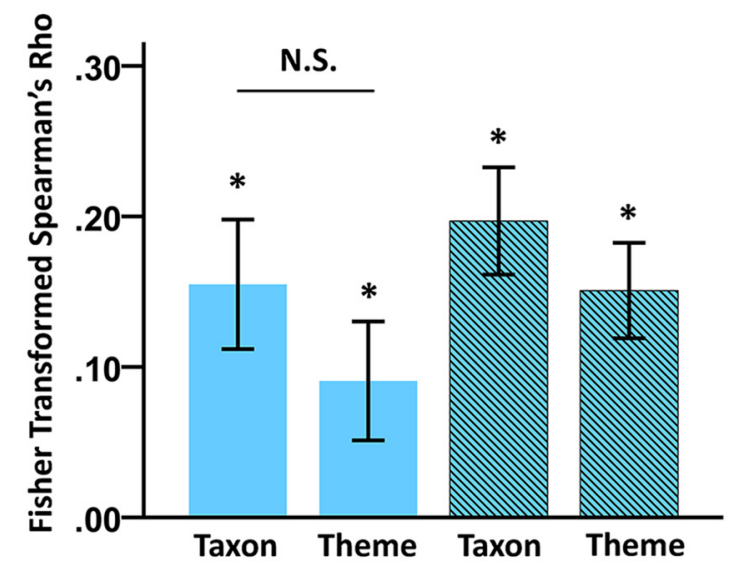

E

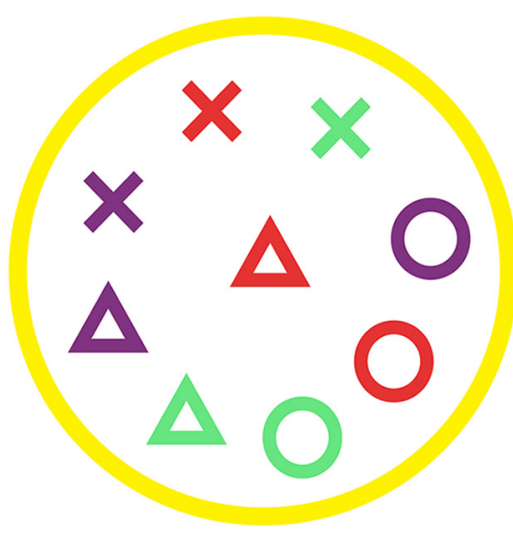

B
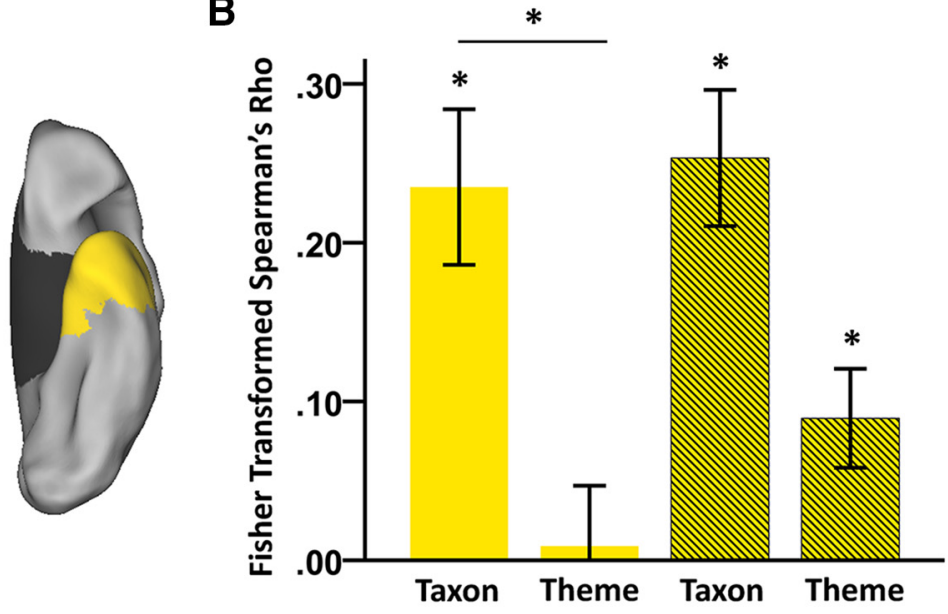

D

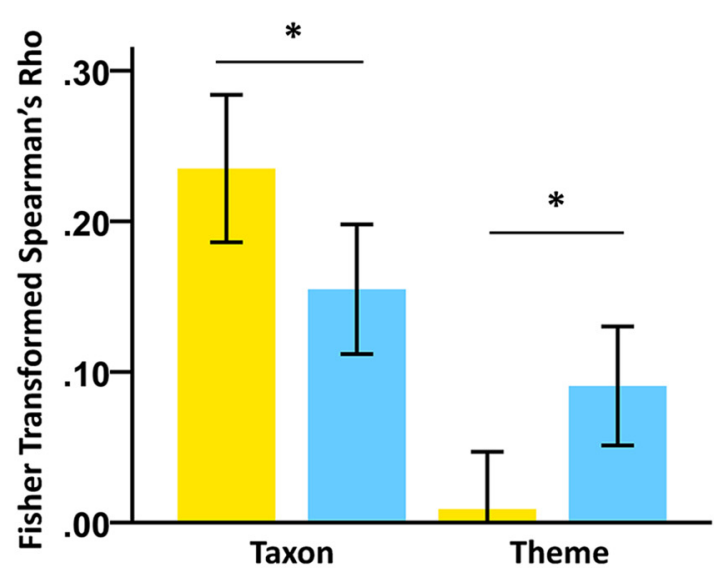

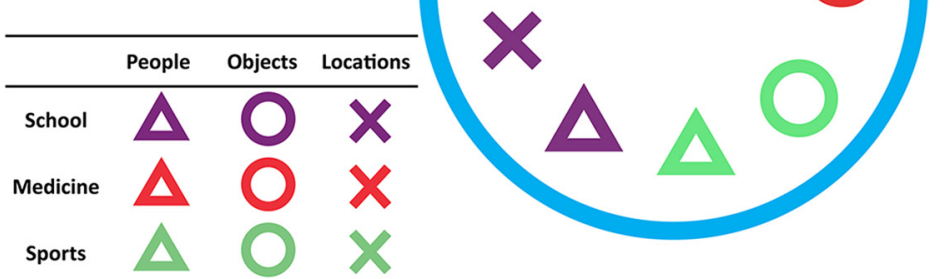

Figure 2. Representational patterns in the left ATL and the left TPJ. $A$, Spatial layout of the left ATL (yellow) and the left TPJ (cyan). The areas were anatomically defined according to the Harvard-0xford atlas. $\boldsymbol{B}$, RSA results in the left ATL. C, RSA results in the left TPJ. D, Direct comparison of the RSA results in the left ATL and those in the left TPJ. In $\boldsymbol{B}-\boldsymbol{D}$, bars with solid colors indicate the Fisher-transformed Spearman's rank correlation between the representational patterns and the taxonomic RDM or the thematic RDM. Bars with stripes indicate the Fisher-transformed Spearman's rank partial correlation between the neural representational patterns and the taxonomic RDM or the thematic RDM after controlling for the thematic or taxonomic differences. ${ }^{*} p<$ 0.05. Error bar indicates \pm SE. E, MDS results of the representational patterns in the left ATL (yellow border) and the left TPJ (the cyan border). Different shapes indicate different taxonomic categories and different colors indicate different themes.

$\left(\right.$ mean ATL $=22.64$, mean TPJ $=44.85$; paired $t_{(18)}=31.3, p<$ 0.001 ), such a tSNR difference could not explain the interaction effect between semantic relations and regions. That is, it is not clear why low tSNR in the ATL would heighten the sensitivity to detect one type of semantic relation and compromise the other.

\section{Whole-brain searchlight}

To explore the effects of brain regions beyond the left ATL and the left TPJ, we implemented a whole-brain RSA-based searchlight analysis (Kriegeskorte et al., 2006) to uncover any brain areas with a representational pattern that significantly correlated with the taxonomic or thematic RDM (Fig. 4). 
A

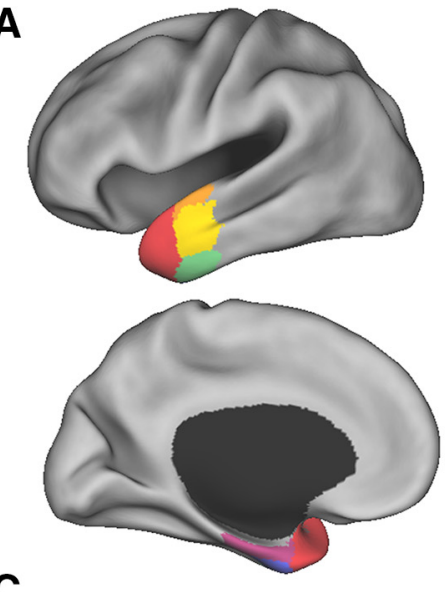

C

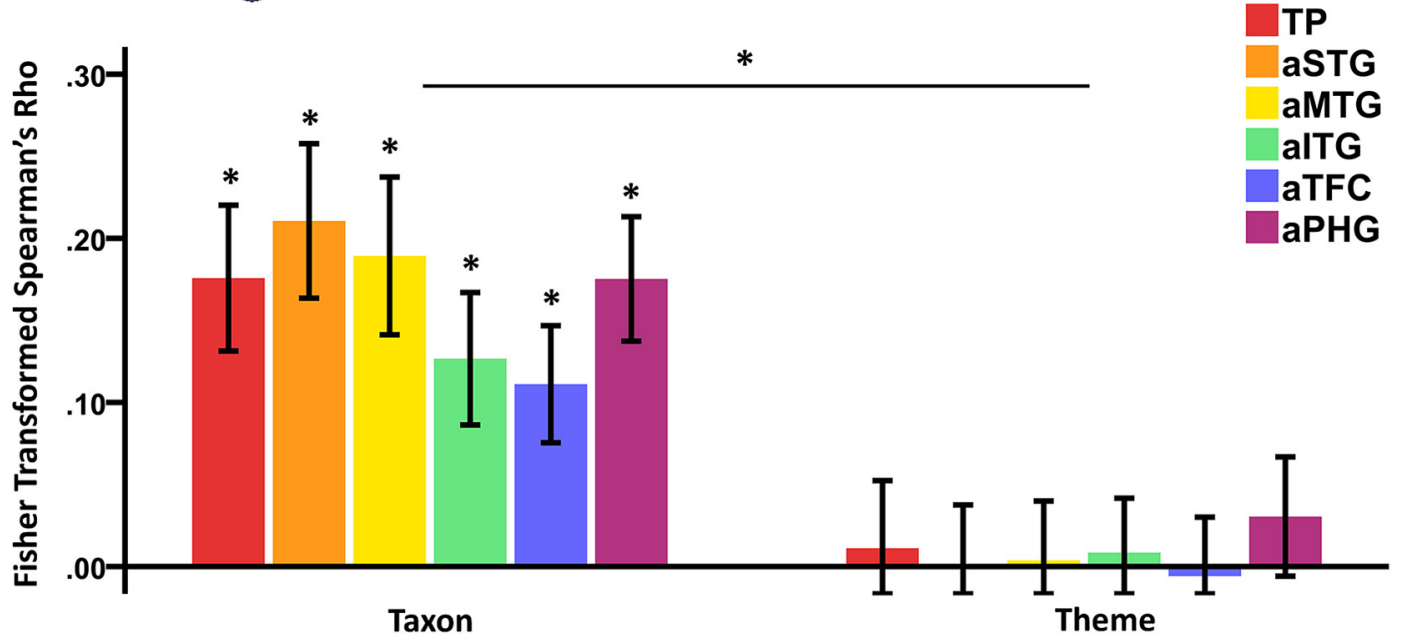

B
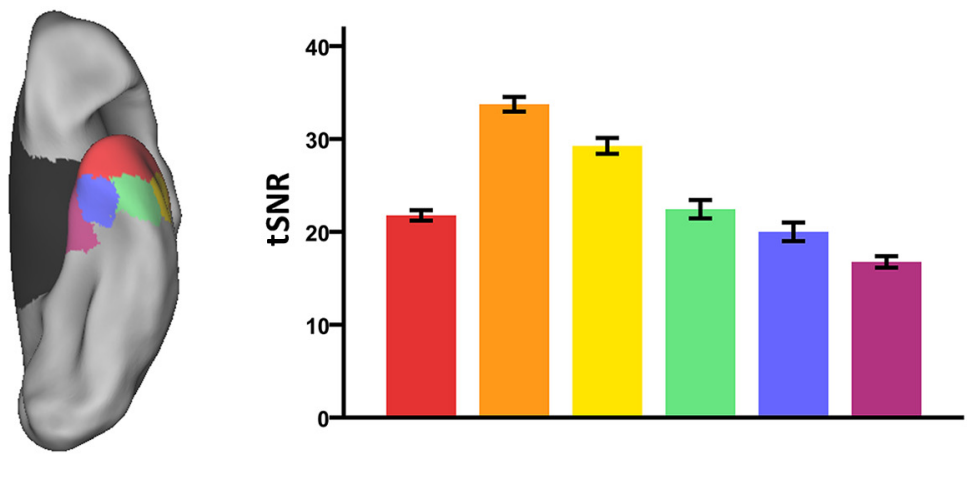

D

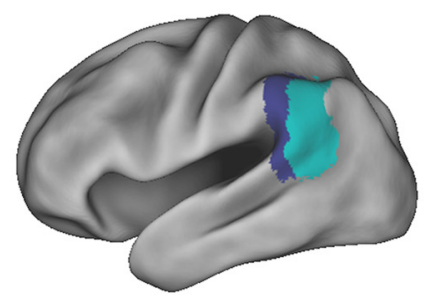

$E$

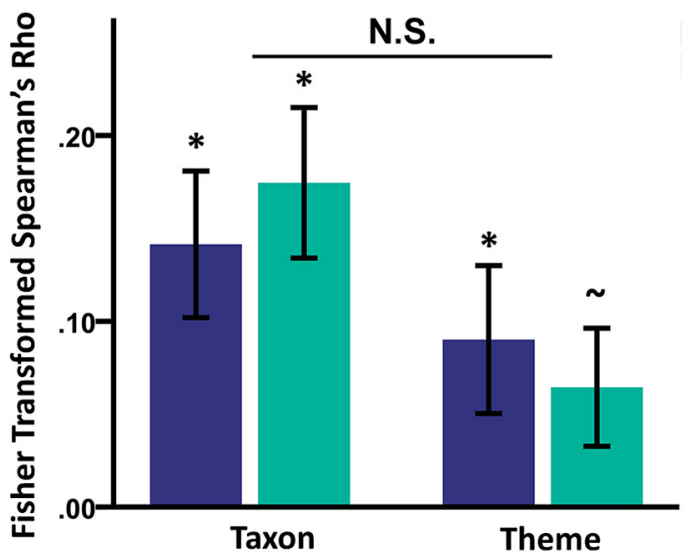

pSMG

AG

Figure 3. Representational pattern in different subregions in the left ATL and TPJ. $A$, Spatial layout of the different subregions in the left ATL. The regions were anatomically defined according to the Harvard-Oxford atlas. B, tSNR of different subregions in the left ATL. C, RSA results in different subregions in the left ATL. ${ }^{*} p<0.05$, Bonferroni corrected. Error bar indicates \pm SE. D, Spatial layout of the different subregions in the left TPJ. The regions were anatomically defined according to the Harvard-0xford atlas. E, RSA results in different subregions in the left TPJ. Error bar indicates \pm SE. ${ }^{*} p<0.05 ; \sim p=0.057$.

\section{Button press}

As participants pressed different buttons according to the taxonomic or thematic tasks in the scanner, the effect of button press inevitably confounded with the taxonomic effect in the taxonomic task (i.e., button press fingers aligned with taxonomic conditions) and the thematic effects in the thematic task (i.e., button press fingers aligned with thematic conditions). To exclude this confounding factor, we first specified what regions associated with the button press by looking at the RSA results with the button press RDMs that were common to the two tasks (see Materials and Methods). At the conventional threshold (primary voxel-level threshold $p<0.001$ and cluster-level $p_{\text {FWE-Corr }}<0.05$ ), we found that the button press effect was confined in the primary motor cortex, the primary somatosensory cortex, and the secondary somatosensory cortex. These areas are outlined with black contours in Figure 4. In the following analyses, clusters showing semanticrelation effects (taxonomic or thematic) that overlapped with these regions would not be considered. 
A

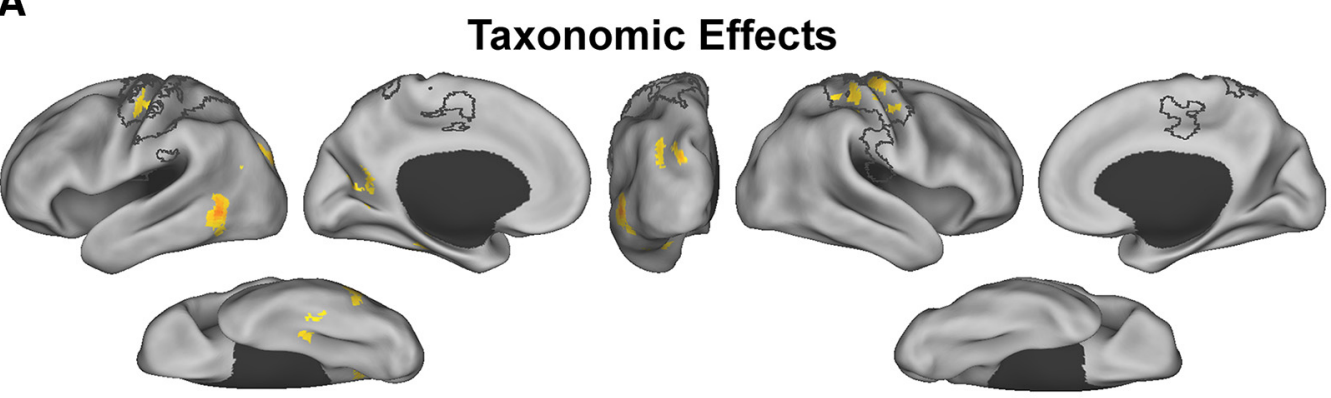

B

Taxonomic Effects When Thematic Difference Was Controlled for
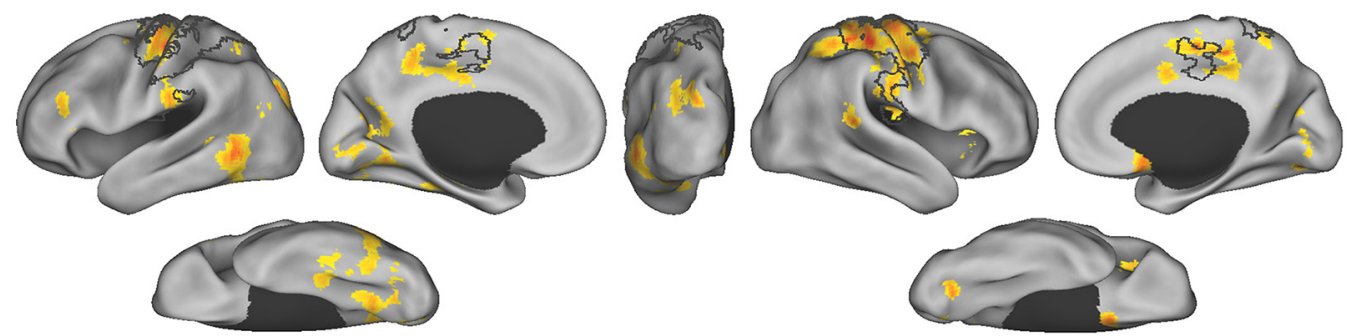

C

Thematic Effects
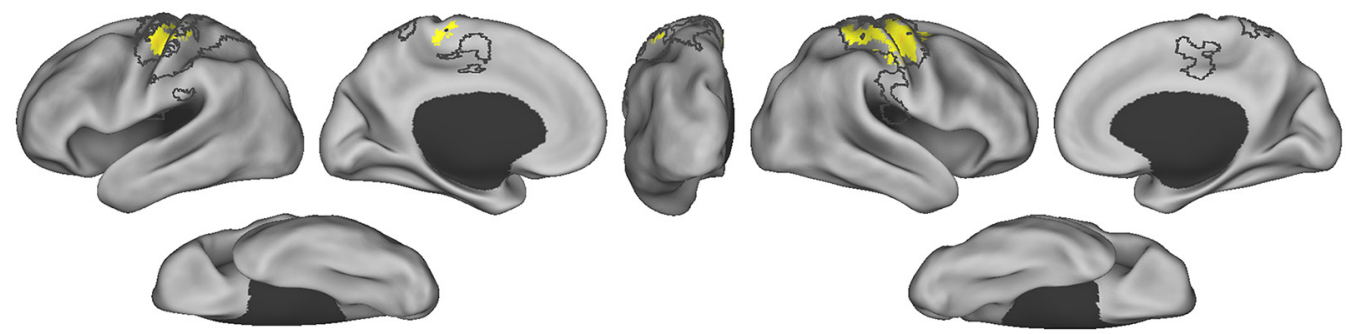

D

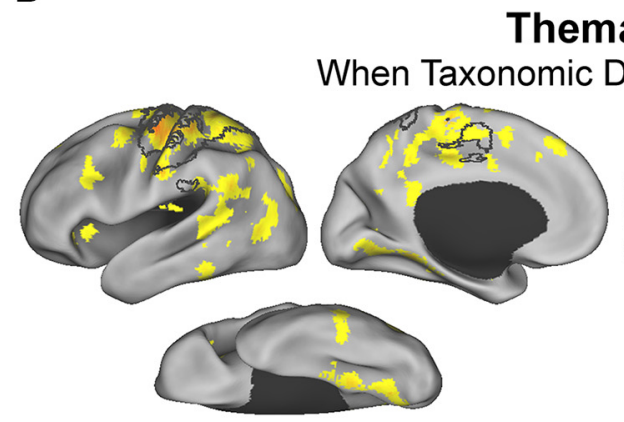

Thematic Effects
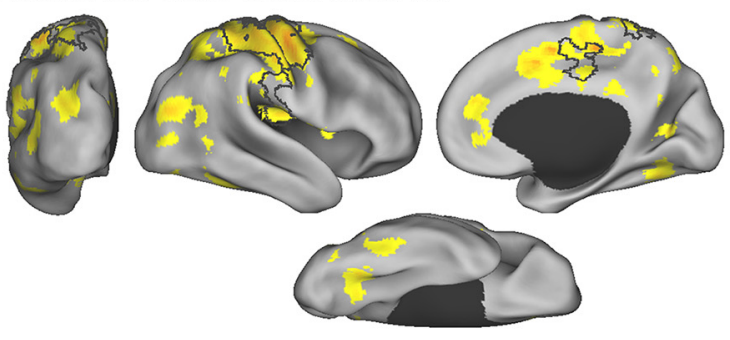

$\mathbf{T}$

3.6

Figure 4. Whole-brain searchlight results. $\boldsymbol{A}$, Taxonomic effects (at a more stringent threshold). Voxel-level $p_{\mathrm{FWE}-\text {-orr }}<0.05$; clusters with sizes smaller than $1000 \mathrm{~mm}^{3}$ are not shown. $\boldsymbol{B}$, Taxonomic effects after controlling for the thematic differences (at a more stringent threshold). Voxel-level $p_{\mathrm{FWE}}$-Corr $<0.05$; clusters with sizes smaller than $1000 \mathrm{~mm}^{3}$ are not shown. $\boldsymbol{C}$, Thematic effects. Primary voxel-level threshold $p<0.001$ and cluster-level $p_{\text {FWE-Corr }}<0.05$. D, Thematic effects after controlling for the taxonomic differences. Primary voxel-level threshold $p<0.001$ and cluster-level $p_{\mathrm{FWE}-\text {-orr }}<0.05$. The areas that outlined in black line contours were associated with button press (primary voxel-level threshold $p<0.001$ and cluster-level $p_{\mathrm{FWE}}$-Corr $<0.05$ ).

Distributed brain regions representing taxonomic information Under the conventional threshold (primary voxel-level threshold $p<0.001$ and cluster-level $\left.p_{\text {FwE-Corr }}<0.05\right)$, the effects of taxonomy were extremely robust, covering much of the temporal, parietal, frontal, and occipital cortex. We thus raised the threshold to a more stringent one (voxel-level $p_{\text {FWE-Corr }}<0.05$, clusters size $>1000 \mathrm{~mm}^{3}$ ). As shown in Figure $4 A$ (see also Table 2), the neural activity pattern in distributed left-hemispheric areas significantly associated with the taxonomic RDM, including the temporooccipital part of the middle and inferior temporal gyrus that extended to the inferior part of the lateral occipital cortex, the superior division of the lateral occipital cortex centered in the transverse occipital sulcus, the posterior division of the fusiform gyrus, and the precuneus region located between the calcarine 
Table 2. Whole-brain searchlight results of the taxonomic effects (voxel-level $\mathrm{p}_{\mathrm{FwE}-\text {-Corr }}<0.05$, clusters with sizes $>1000 \mathrm{~mm}^{3}$ )

\begin{tabular}{|c|c|c|c|c|c|c|c|}
\hline \multirow{2}{*}{\multicolumn{2}{|c|}{ Anatomical label }} & \multirow{3}{*}{$\begin{array}{l}\text { Peak voxel ( } t \text { value) } \\
10.65\end{array}$} & \multirow{3}{*}{$\begin{array}{l}\text { Cluster size (voxels) } \\
419\end{array}$} & \multirow{3}{*}{$\frac{p_{\text {FWE-Corr }} \text { (voxel level) }}{0.0001}$} & \multicolumn{3}{|c|}{ MNI coordinates } \\
\hline & & & & & \multirow{2}{*}{$\frac{x}{-54}$} & \multirow{2}{*}{$\frac{Y}{-60}$} & \multirow{2}{*}{$\frac{Z}{-2}$} \\
\hline L & $\begin{array}{l}\text { Middle temporal gyrus, temporooccipital part } \\
\text { Lateral occipital cortex, inferior division } \\
\text { Inferior temporal gyrus, temporooccipital part }\end{array}$ & & & & & & \\
\hline L & Lateral occipital cortex, superior division & 7.83 & 230 & 0.0029 & -22 & -86 & 30 \\
\hline L & Temporal fusiform cortex, posterior division & 7.80 & 147 & 0.0030 & -36 & -26 & -20 \\
\hline L & $\begin{array}{l}\text { Precuneous cortex } \\
\text { Intracalcarine cortex }\end{array}$ & 7.69 & 190 & 0.0036 & -8 & -66 & 16 \\
\hline \multicolumn{8}{|c|}{ After controlling for thematic difference } \\
\hline L & $\begin{array}{l}\text { Middle temporal gyrus, temporooccipital part } \\
\text { Lateral occipital cortex, inferior division } \\
\text { Inferior temporal gyrus, temporooccipital part } \\
\text { Temporal occipital fusiform cortex }\end{array}$ & 12.46 & 843 & 0.0002 & -54 & -58 & -4 \\
\hline $\mathrm{R}$ & Subcallosal cortex & 10.45 & 364 & 0.0003 & 8 & 14 & -14 \\
\hline L & Subcallosal cortex & & & & & & \\
\hline $\mathrm{R}$ & Occipital fusiform gyrus & 9.94 & 160 & 0.0003 & 24 & -72 & -8 \\
\hline L & $\begin{array}{l}\text { Intracalcarine cortex } \\
\text { Precuneous cortex } \\
\text { Lingual gyrus } \\
\text { Occipital fusiform gyrus } \\
\text { Cuneal cortex }\end{array}$ & 9.75 & 851 & 0.0005 & -8 & -68 & 16 \\
\hline $\mathrm{R}$ & $\begin{array}{l}\text { Precuneous cortex } \\
\text { Cuneal cortex }\end{array}$ & & & & & & \\
\hline $\mathrm{R}$ & $\begin{array}{l}\text { Insular cortex } \\
\text { Frontal operculum cortex }\end{array}$ & 9.71 & 184 & 0.0005 & 32 & 20 & -4 \\
\hline L & $\begin{array}{l}\text { Lateral occipital cortex, superior division } \\
\text { Occipital pole }\end{array}$ & 9.49 & 407 & 0.0005 & -26 & -80 & 34 \\
\hline L & $\begin{array}{l}\text { Middle frontal gyrus } \\
\text { Superior frontal gyrus }\end{array}$ & 9.27 & 126 & 0.0007 & -28 & 4 & 54 \\
\hline L & $\begin{array}{l}\text { Temporal fusiform cortex, posterior division } \\
\text { Hippocampus } \\
\text { Parahippocampal gyrus, posterior division }\end{array}$ & 8.12 & 323 & 0.0019 & -38 & -28 & -18 \\
\hline $\mathrm{L}$ & $\begin{array}{l}\text { Middle frontal gyrus } \\
\text { Inferior frontal gyrus, pars triangularis }\end{array}$ & 8.05 & 130 & 0.0020 & -46 & 36 & 18 \\
\hline $\mathrm{L}$ & $\begin{array}{l}\text { Lateral occipital cortex, superior division } \\
\text { Superior parietal lobule }\end{array}$ & 7.85 & 204 & 0.0024 & -24 & -62 & 44 \\
\hline L & $\begin{array}{l}\text { Intracalcarine cortex } \\
\text { Lingual gyrus }\end{array}$ & 7.43 & 260 & 0.0041 & -2 & -84 & 2 \\
\hline $\mathrm{R}$ & $\begin{array}{l}\text { Intracalcarine cortex } \\
\text { Lingual gyrus }\end{array}$ & & & & & & \\
\hline
\end{tabular}

Clusters that overlapped with the regions associated with the button-press effect were excluded. Regions are labeled according to the Harvard-0xford cortical and subcortical atlas.

cortex and the posterior cingulate gyrus. After controlling for the thematic difference using Spearman's rank partial correlation, the taxonomic effect became stronger in similar regions (higher voxel $t$-values and larger clusters; Fig. $4 B$, Table 2, voxel-level $p_{\text {FWE-Corr }}<0.05$ and clusters size $>1000 \mathrm{~mm}^{3}$ ) and additionally included bilateral subcallosal cortices, bilateral intracalcarine cortices that extended to lingual gyri, the left middle frontal gyrus that extended to the superior frontal gyrus, the left middle frontal gyrus that extended to the triangular part of the inferior frontal gyrus, the right anterior insula cortex that extended to the frontal operculum cortex, the right occipital-fusiform gyrus, and the left superior division of the lateral occipital cortex that extended to the superior parietal lobule.

Regions that represented thematic information emerge only after controlling for the taxonomic difference

Correlating the neural activity pattern with the thematic RDM in the whole-brain searchlight yielded significant clusters only in the areas associated with button press effects at the same threshold (Fig. 4C; primary voxel-level threshold $p<0.001$ and clusterlevel $\left.p_{\text {FWE-Corr }}<0.05\right)$. After controlling for the taxonomic RDM, significant thematic effects emerged in widely distributed regions across the occipital, frontal, temporal, and parietal corti- ces (Fig. 4D, Table 3; primary voxel-level threshold $p<0.001$ and cluster-level $\left.p_{\text {FWE-Corr }}<0.05\right)$.

Taxonomic and thematic representation at the semantic subnetwork level

Beyond the regional level, we examined the representational patterns in regions that formed two topologically dissociable semantic subnetworks, the semantic-DMN subnetwork and the semantic-PSN subnetwork (Xu et al., 2016) (Fig. 5A).

As shown in Figure $5 B$, the RSA result showed that the activity pattern in the semantic-DMN subnetwork significantly correlated with the taxonomic RDM (mean $r=0.24 ; t_{(18)}=5.48, p<$ 0.001 ) and not with the thematic RDM (mean $r=0.03, t_{(18)}=$ $1.00, p=0.332$ ), with significant differences between these two effects (paired $t_{(18)}=3.362, p=0.003$ ). After controlling for the thematic difference, the activity pattern in the semantic-DMN subnetwork remained significantly correlated with the taxonomic RDM (mean $r=0.27$, partial correlation, $t_{(18)}=6.292$, $p<0.001)$ and, after controlling for the taxonomic difference, became significantly correlated with the thematic RDM (partial correlation, mean $r=0.12, t_{(18)}=4.281, p<0.001$ ). These results indicated that the activity pattern in the semantic-DMN 
Table 3. Whole-brain searchlight results of the thematic effects (primary voxel-level threshold $p<0.001$ and cluster-level $p_{\text {FwE-Corr }}<0.05$ )

\begin{tabular}{|c|c|c|c|c|c|c|c|}
\hline \multirow{2}{*}{\multicolumn{2}{|c|}{ Anatomical label }} & \multirow[b]{2}{*}{ Peak voxel ( $t$ value) } & \multirow[b]{2}{*}{ Cluster size (voxels) } & \multirow[b]{2}{*}{$p_{\text {FWE-corr }}$ (cluster level) } & \multicolumn{3}{|c|}{ MNI coordinates } \\
\hline & & & & & $x$ & Y & Z \\
\hline \multicolumn{8}{|c|}{ None } \\
\hline \multicolumn{8}{|c|}{ After controlling for taxonomic difference } \\
\hline $\mathrm{R}$ & $\begin{array}{l}\text { Lateral occipital cortex, superior division } \\
\text { Lateral occipital cortex, inferior division } \\
\text { Middle temporal gyrus, temporooccipital part }\end{array}$ & 7.72 & 760 & 0.0023 & 42 & -74 & 20 \\
\hline $\mathrm{L}$ & $\begin{array}{l}\text { Middle frontal gyrus } \\
\text { Inferior frontal gyrus, pars opercularis }\end{array}$ & 6.67 & 229 & 0.0148 & -40 & 18 & 26 \\
\hline$L$ & $\begin{array}{l}\text { Angular gyrus } \\
\text { Supramarginal gyrus, posterior division } \\
\text { Lateral occipital cortex, inferior division } \\
\text { Lateral occipital cortex, superior division } \\
\text { Middle temporal gyrus, temporooccipital part } \\
\text { Superior temporal gyrus, posterior division } \\
\text { Middle temporal gyrus, posterior division } \\
\text { Parietal operculum cortex }\end{array}$ & 6.57 & 1301 & 0.0012 & -56 & -56 & 30 \\
\hline $\mathrm{L}$ & $\begin{array}{l}\text { Lingual gyrus } \\
\text { Parahippocampal gyrus, posterior division }\end{array}$ & 6.51 & 438 & 0.0038 & -20 & -46 & -12 \\
\hline $\mathrm{R}$ & $\begin{array}{l}\text { Precuneous cortex } \\
\text { Lateral occipital cortex, superior division }\end{array}$ & 6.50 & 148 & 0.0322 & 12 & -64 & 48 \\
\hline $\mathrm{R}$ & $\begin{array}{l}\text { Central opercular cortex } \\
\text { Putamen } \\
\text { Amygdala } \\
\text { Insular cortex }\end{array}$ & 6.43 & 460 & 0.0035 & 36 & 8 & 14 \\
\hline R & $\begin{array}{l}\text { Paracingulate gyrus } \\
\text { Cingulate gyrus, anterior division }\end{array}$ & 6.43 & 306 & 0.0081 & 14 & 44 & 20 \\
\hline $\mathrm{R}$ & $\begin{array}{l}\text { Intracalcarine cortex } \\
\text { Precuneous cortex }\end{array}$ & 6.40 & 312 & 0.0077 & 18 & -64 & 10 \\
\hline L & Precuneous cortex & & & & & & \\
\hline $\mathrm{L}$ & Frontal pole & 6.36 & 133 & 0.0376 & -36 & 44 & 14 \\
\hline $\mathrm{R}$ & Superior frontal gyrus & 6.30 & 248 & 0.0125 & 18 & 16 & 50 \\
\hline $\mathrm{L}$ & $\begin{array}{l}\text { Frontal orbital cortex } \\
\text { Insular cortex }\end{array}$ & 6.07 & 195 & 0.0197 & -36 & 26 & -4 \\
\hline R & $\begin{array}{l}\text { Temporal occipital fusiform cortex } \\
\text { Inferior temporal gyrus, temporooccipital part } \\
\text { 0ccipital fusiform gyrus } \\
\text { Lingual gyrus } \\
\text { Lateral occipital cortex, inferior division }\end{array}$ & 6.03 & 628 & 0.0025 & 30 & -60 & -16 \\
\hline L & Thalamus & 5.98 & 153 & 0.0308 & -12 & -28 & 8 \\
\hline $\mathrm{L}$ & $\begin{array}{l}\text { Inferior temporal gyrus, posterior division } \\
\text { Inferior temporal gyrus, temporooccipital part }\end{array}$ & 5.85 & 239 & 0.0136 & -56 & -42 & -18 \\
\hline $\mathrm{L}$ & Lateral occipital cortex, superior division & 5.84 & 317 & 0.0074 & -30 & -82 & 26 \\
\hline L & Superior frontal gyrus & 5.71 & 179 & 0.0232 & -4 & 40 & 46 \\
\hline L & $\begin{array}{l}\text { Amygdala } \\
\text { Hippocampus }\end{array}$ & 5.46 & 197 & 0.0194 & -26 & -6 & -22 \\
\hline $\begin{array}{l}L \\
R\end{array}$ & $\begin{array}{l}\text { Cingulate gyrus, posterior division } \\
\text { Cinqulate gyrus, posterior division }\end{array}$ & 5.34 & 211 & 0.0166 & -2 & -48 & 26 \\
\hline
\end{tabular}

Clusters that overlapped with the regions associated with the button-press effect were excluded. Regions are labeled according to the Harvard-0xford cortical and subcortical atlas.

subnetwork was primarily organized by taxonomic information and secondarily organized by thematic information.

As shown in Figure $5 C$, the RSA results of the semantic-PSN subnetwork was similar to that in the semantic-DMN subnetwork. The neural activity pattern in the semantic-PSN subnetwork significantly correlated with the taxonomic RDM (mean $\left.r=0.25 ; t_{(18)}=4.94, p<0.001\right)$ and not with the thematic RDM (mean $r=0.04 ; t_{(18)}=1.26, p=0.223$ ), with significant difference between the strengths of these two effects (paired $t_{(18)}=$ $2.66, p=0.016$ ). After controlling for the thematic difference, the taxonomic effects remained significant (mean $r=0.28$; partial correlation, $\left.t_{(18)}=6.08, p<0.001\right)$ and, after controlling for the taxonomic difference, the thematic effects also became significant (partial correlation, mean $r=0.13 ; t_{(18)}=4.88, p<0.001$ ).

When comparing directly the amount of taxonomic and thematic information carried by these two subnetworks (Fig. 5D) using a two (DMN vs PSN semantic subnetworks) $\times$ two (taxonomic vs thematic information) repeated-measures ANOVA, a significant main effect was observed only for the semantic relations $\left(F_{(1,18)}=10.15, p=0.005\right)$. There was neither a main effect of semantic subnetworks $\left(F_{(1,18)}=0.52, p=0.481\right)$ nor an interaction $\left(F_{(1,18)}=0.02, p=0.884\right)$, suggesting the similar representational structure in terms of taxonomic and thematic dimensions in these two subnetworks.

\section{Modulation effects by the task goals}

Finally, we assessed the degree to which the activity pattern in these semantic-related areas (left ATL, left TPJ, semantic-DMN subnetwork, and semantic-PSN subnetwork) changed according to different semantic tasks (Fig. 6A). A two (taxonomic vs thematic judgment tasks) $\times$ two (taxonomic vs thematic information) repeated-measures ANOVA revealed no significant effects 
A
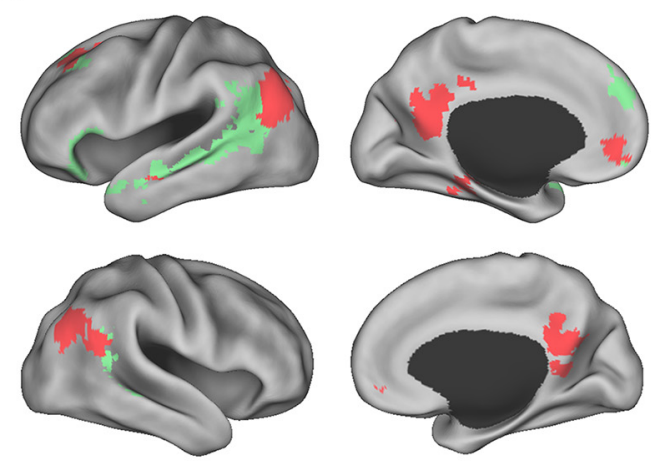

\section{C}

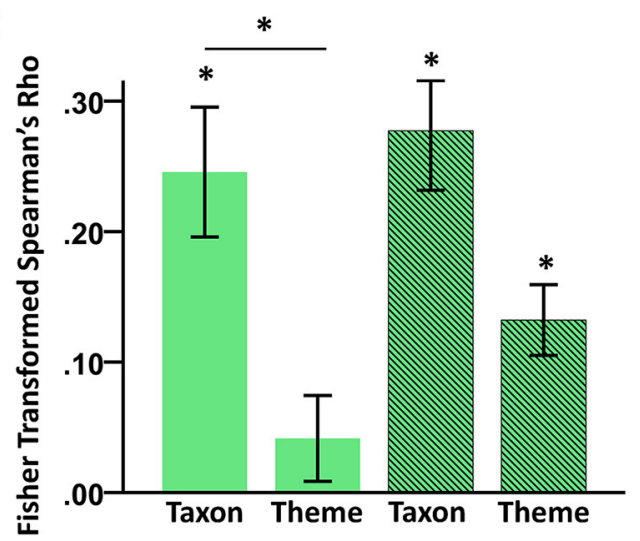

B

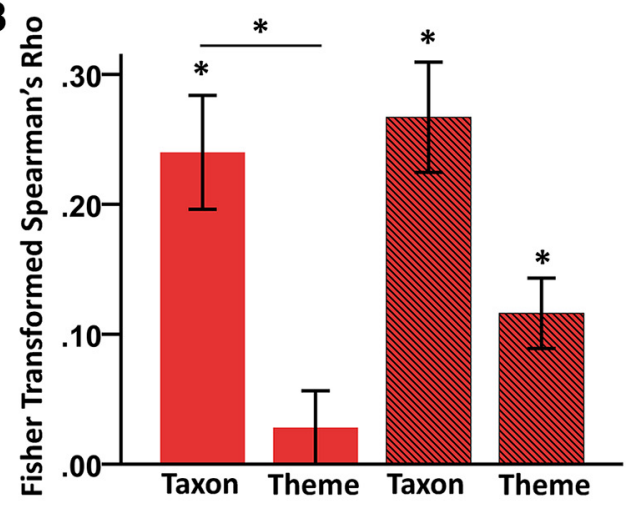

D

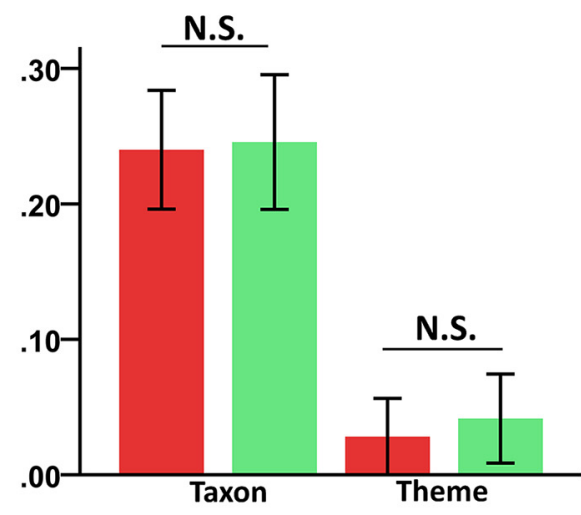

Figure 5. Representational pattern of the semantic-DMN subnetwork and the semantic-PSN subnetwork. $A$, Spatial layout of the DMN subnetwork (red) and the PSN subnetwork (green). These semantic subnetworks were defined by Xu et al. (2016). B, RSA results of the semantic-DMN subnetwork. C, RSA results of the semantic-PSN subnetwork. $\boldsymbol{D}$, Direct comparison of the RSA results of the semantic-DMN subnetwork and those of the semantic-PSN subnetwork. Bars with solid colors indicate the Fisher-transformed Spearman's rank correlation between the neural representational patterns and the taxonomic RDM or the thematic RDM. Bars with stripes indicate the Fisher-transformed Spearman's rank partial correlation between the neural representational patterns and the taxonomic RDM or the thematic RDM after controlling for the other dimension. ${ }^{*} p<0.05$. N.S., Not significant. Error bar indicates \pm SE.

of semantic tasks ( $p \geq 0.352$ ), a significant main effect of semantic relation type in the left ATL, the semantic-DMN subnetwork and the semantic-PSN subnetwork $(p \leq 0.013$; marginally significant in the left TPJ, $\left.F_{(1,18)}=3.26, p=0.088\right)$, and a significant interaction in all regions/systems $(p \leq 0.001)$. The post hoc analyses revealed that, in all regions, the taxonomic effects were stronger in the taxonomic judgment task than the thematic judgment task (paired $t$ test, $p \leq 0.002$ ) and the thematic effects were stronger in the thematic judgment task than the taxonomic judgment task (paired $t$ test, $p \leq 0.013$ ).

\section{Validation analyses}

We performed four validation analyses to exclude the potential effects of confounding factors: (1) words in the thematic category of sports tended to have lower prototypicality and were associated with a broader domain (see Materials and Methods), so we excluded the thematic category of sports and repeated the ROI and subnetwork analyses; (2) we further excluded the behavioral accuracy differences by repeating all of the ROI and subnetwork analyses using the Spearman partial correlation and included the accuracy RDMs of individual participants as covariate; (3) we further regressed out two control matrices (the word frequency and the visual similarity matrices; see Materials and Methods) in the RSA; and (4) in another attempt to fully exclude the confounding effects of button press in the whole-brain searchlight analyses, we performed an additional analysis by correlating the taxonomic RDM with the neural activity pattern in the thematic task (i.e., button press fingers aligned with thematic conditions) and correlating the thematic RDM with the neural activity pattern in the taxonomic task (i.e., button press fingers aligned with taxonomic conditions). In the ROI and subnetwork analyses, the result patterns in all these validation analyses were similar to those in the main analysis. In the whole-brain searchlight analyses, the taxonomic effects (in the thematic judgment task) were mainly found in the left temporooccipital part of the inferior and middle temporal gyrus, the precuneus, and the right anterior medial temporal lobe (primary voxel-level threshold $p<0.001$ and cluster-level $\left.p_{\text {FWE-Corr }}<0.05\right)$. These effects became stronger and involved more regions when the thematic differences were controlled for. The thematic effects (in the taxonomic judgment task) only appeared when the taxonomic differences were controlled for, which mainly fell in bilateral superior occipital gyri, bilateral TPJs, the right superior and middle frontal gyrus, the precuneus, the anterior cingulate and the adjacent paracingulate gyrus, the left posterior parahippocampal gyrus, and the ventral occipital/lingual/fusiform gyri $(p<0.001$, uncorrected, cluster size $>20$ voxels). Overall, both the taxonomic-specific effects and the thematic-specific effects fell into similar regions as those reported in the main results.

\section{Discussion}

Using the RSA, we elucidated the brain regions in which words were organized along taxonomic ("doctor" and "teacher" closer) or thematic ("doctor" and "stethoscope" closer) dimensions. As summarized in Table 4, we found a left-lateralized distributed network that primarily respected words' taxonomic structures. 
A
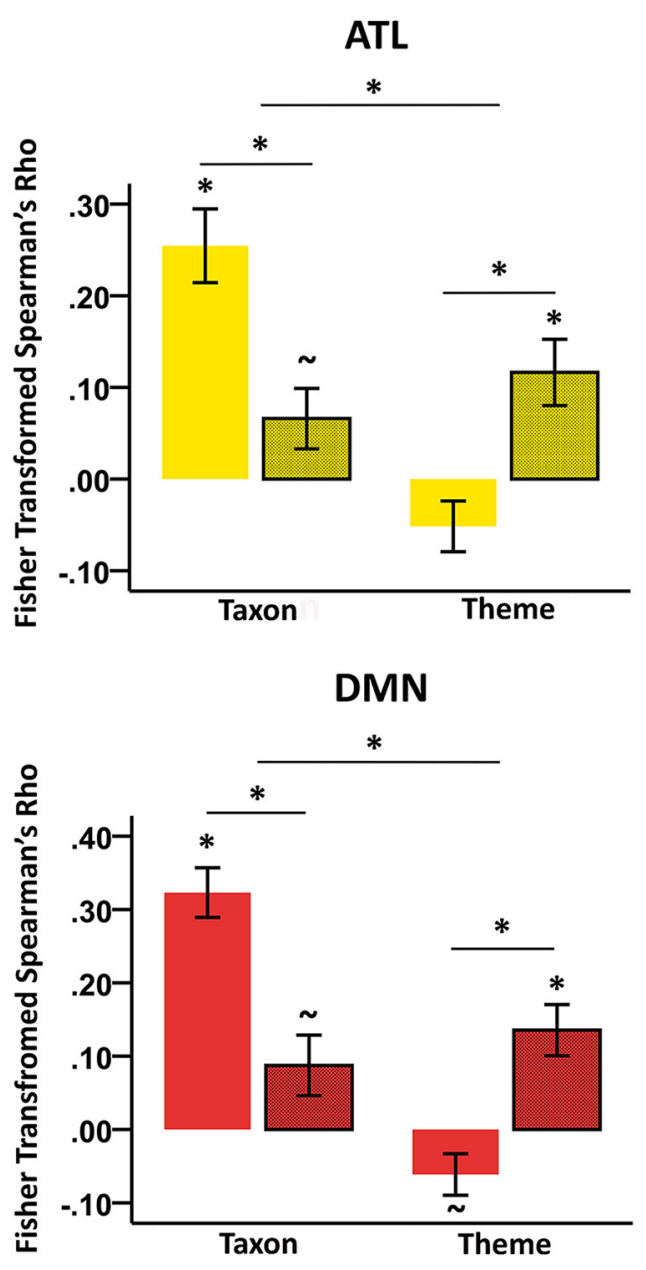

B
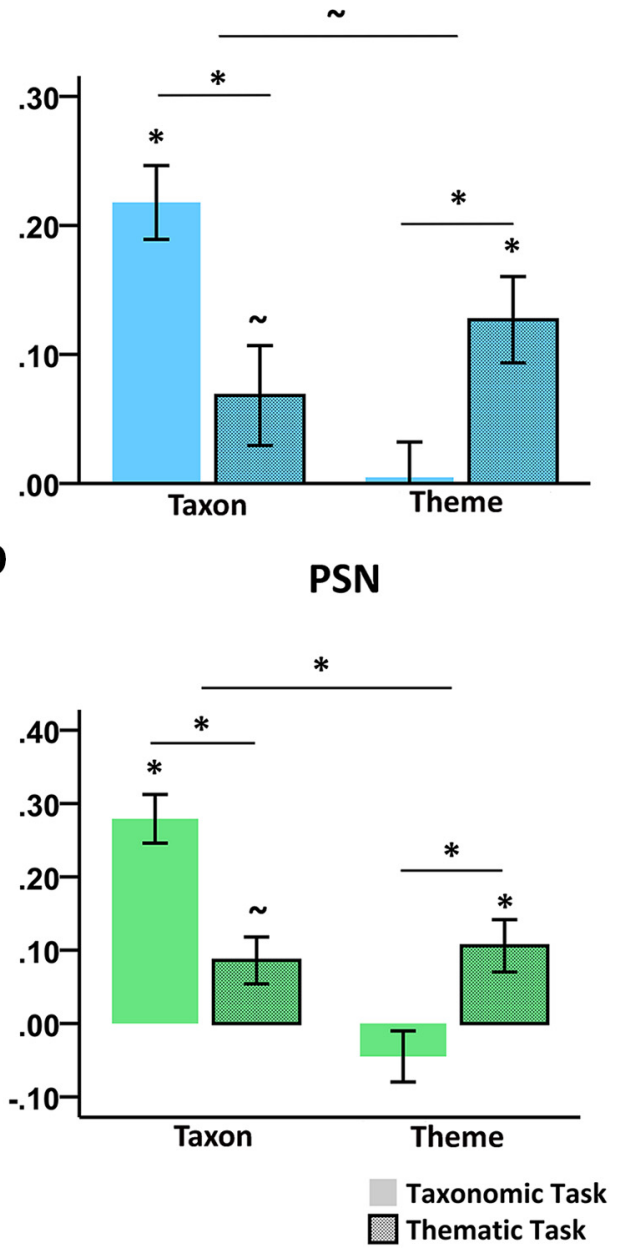

Figure 6. Task modulation effects of neural representational patterns. The Fisher-transformed Spearman's rank correlations between the representational patterns in each neural substrate and the taxonomic RDM or the thematic RDM under different semantic judgment tasks are illustrated. Bars with solid colors indicate taxonomic tasks. Bars with borders and dots indicate thematic tasks. $\sim p<0.1$ or $p<0.05$ (uncorrected); ${ }^{*} p<0.05$ (Bonferroni corrected, when a multiple-comparison correction was needed). Error bar indicates \pm SE.

Table 4. Schematic result summary

\begin{tabular}{|c|c|c|c|c|c|}
\hline & Taxonomic effects & Thematic effects & $\begin{array}{l}\text { Taxonomic effects after } \\
\text { controlling for thematic } \\
\text { difference }\end{array}$ & $\begin{array}{l}\text { Thematic effects after } \\
\text { controlling for taxonomic } \\
\text { difference }\end{array}$ & $\begin{array}{l}\text { Modulated by } \\
\text { task demands }\end{array}$ \\
\hline \multicolumn{6}{|l|}{ ROls } \\
\hline TPJ & +++ & ++ & +++++ & +++ & V \\
\hline $\begin{array}{l}\text { Whole-brain } \\
\text { searchlight }\end{array}$ & $\begin{array}{c}++++ \\
\text { ITG/MTG/L0, TOS, } \\
\text { precuneus, fusiform } \\
\text { (see Table 2) }\end{array}$ & - & $\begin{array}{l}++++ \\
\text { ITG/MTG/L0, TOS, precuneus, } \\
\text { fusiform, and other brain } \\
\text { areas (see Table 2) }\end{array}$ & $\begin{array}{c}++ \\
\text { L0, PT0, and other } \\
\text { brain areas } \\
\text { (see Table 3) }\end{array}$ & not tested \\
\hline \multicolumn{6}{|c|}{ Semantic subnetworks } \\
\hline DMN & ++++ & - & +++++ & ++ & V \\
\hline PSN & ++++ & - & +++++ & ++ & V \\
\hline
\end{tabular}

ITG, Inferior temporal gyrus; MTG, middle temporal gyrus; L0, lateral occipital; TOS, transverse occipital sulcus; PTO, parietal-temporal-occipital association areas.

${ }^{a}$ Number of plus signs indicates relative strength.

This network mainly included the ATL, the TPJ, the temporooccipital part of the middle and inferior temporal gyrus that extended to the inferior part of the lateral occipital cortex, the superior division of the lateral occipital cortex centered in the transverse occipital sulcus, the posterior division of the temporal fusiform cortex, and the precuneus cortex. In contrast, the effects of thematic relations were directly observed only in the left TPJ in the ROI analysis and emerged in many other regions, including the ATL, after the taxonomic difference was controlled for. The same pattern was observed when looking at a larger system level: 
the neural response pattern of each of the two semantic subnetworks was primarily associated with the taxonomic RDM and showed association with the thematic RDM after the taxonomic difference was controlled for. That is, the primary organization dimension for concepts appears to be taxonomic categories, with thematic categories only embedded within the taxonomic structure.

The regions showing effects of taxonomic organization corresponded well to studies in which such taxonomic dimension was examined using MVPA/RSA (Shinkareva et al., 2011; Devereux et al., 2013; Fairhall and Caramazza, 2013b; Clarke and Tyler, 2014; Simanova et al., 2014) and were also consistent with the vast literature showing taxonomic-category-preferring activities using univariate approaches for the three taxonomic categories used here, people, manmade objects, and locations (Binder et al., 2009; Fairhall and Caramazza, 2013a). The observations that the left TPJ, and not the left ATL, primarily showed a significant correlation with the thematic organization, is consistent with the neuropsychological studies showing that lesions in the left TPJ were relatively specifically associated with thematic errors (Schwartz et al., 2011).

Different from the previous studies, however, we found that the activity patterns in regions that are classically viewed to respect taxonomic categories, for example, the left ATL, the left transverse occipital sulcus, and the precuneus, actually further respect the thematic dimension once the taxonomic difference was controlled for. That is, thematic relations appear to be embedded within the taxonomic structure rather than being represented by separate brain regions. The concepts are first organized by taxonomic categories of people, manmade objects, and locations. In addition, across different taxonomic categories, there are further effects of thematic association; "doctor" and "stethoscope" are represented in a more similar pattern than "doctor" and "chalk." On a larger scale, two semantic subsystems that were parcellated based on modularity structures of the resting-state semantic network (Xu et al., 2016) were examined. They encompassed brain regions that tend to be more relevant for multimodal experiential and language-supported semantic encoding, respectively (Xu et al., 2017). The results showed that, in both subsystems, taxonomic information was the primary dimension and thematic relations were further embedded within the taxonomic structure. The exact mechanisms for both systems in respecting primarily the taxonomic relation remain to be further explained.

Cognitively, taxonomic and thematic relations have been hypothesized to reflect different kinds of representational mechanisms (Estes et al., 2011). The taxonomic relation is primarily based on the similarity of semantic features (e.g., various types of sensorimotor properties) and the thematic relation is based on the co-occurrence and complementary relations in the same event. Our findings that most regions represented both types of semantic relations suggest that there might be a general neural representational mechanism underlying both taxonomic and thematic relations (see discussions in Jackson et al., 2015). One possibility is that the thematic cooccurrence is one type of "semantic feature" or results from the integration of several particular types of semantic features, such as space, time, and action (see below). Because the neural representational pattern may reflect the overall similarity of semantic features, the taxonomic relation, which is based on the similarity in the majority of semantic features, appears much stronger than the thematic relation, which is based on the similarity in fewer types of semantic features. Given that space and action are central to forming an event, brain areas relating to spatial and action-related semantic feature processing is likely to be involved in thematic representation. This may explain why the effects of thematic organization are most transparent in the TPJ. The TPJ is centered between the transverse occipital sulcus, which plays an important role in the cognition of scenes (Dilks et al., 2013), and the posterior temporal lobe and the rostral part of the inferior parietal lobe, which are involved in motion and action cognition (Iacoboni and Dapretto, 2006). The area with the strongest thematic effects (after taxonomic differences were controlled for) in the whole-brain searchlight analyses, the right superior lateral occipital cortex that is adjacent to the parietal cortex, is also found to be involved in visuospatial processing (Goodale and Milner, 1992; Mellet et al., 1995). Lesions to the bilateral occipitoparietal lobes lead to simultanagnosia, a syndrome characterized by the restricted attention window to only one object at a time and the failure to comprehend the overall meaning of a scene (Bálint, 1909; Dalrymple et al., 2013). Furthermore, the involvement of the dorsal visual system in thematic processing is consistent with the recent finding in a word-to-picture eye-tracking study, in which earlier activation was found on thematically related objects compared with functionally related items (Kalénine et al., 2012).

It is also worth considering whether the asymmetric organization (the thematic associations are embedded in the taxonomic structures) could also be explained by different manners of neural coding. The taxonomic relation, which entails at least partly integration of sensorimotor features, may rely on the distributed coding of large populations of neurons, a coding paradigm that has been found widely to represent sensorimotor information (Georgopoulos et al., 1986, 1988). Conversely, the thematic relation may be built on the cofiring of concepts with fewer overlapping sensorimotor features in the same time/space sharing event and might be better captured by sparse neuronal coding or associations/connections across the conceptual representations (Binder, 2016). Consistent with these assumptions, it has been reported that the concept cell assemblies in the medial temporal lobe encode semantic associations in a sparse manner (Quiroga, 2012; De Falco et al., 2016). In addition, the MVPA study by Anderson et al. (2014) showed that, when the whole-brain activity pattern was used to predict various concrete concept conditions, the leave-one-domain-out taxonomic predictions had better performances than the leave-one-taxonomic-category-out domain-related predictions, indicating that the taxonomic information was better captured by the whole-brain activity pattern. How such population coding versus sparse coding (or overlapping versus association) of taxonomic versus thematic relations could explain the current findings remains to be further explored.

Our results showed a significant effect of task modulation for both dimensions in our regions/subnetworks of interest, with effects of a semantic dimension heightened in the task judging that dimension compared with the task judging the other dimension. This result is consistent with findings that neural activity patterns may be modulated by task-related attention (Stokes et al., 2009; Chiu et al., 2011; Hjortkjær et al., 2018; Nastase et al., 2017). Regarding semantic processing, however, some previous studies have suggested or theoretically postulated that the neural representation pattern in only the frontoparietal regions (Bracci et al., 2017) or the integrative layer between the semantic control areas and the semantic representational areas (Ralph et al., 2017) are affected by the semantic tasks. The effects and mechanisms of task goal modulation warrant further understanding.

To conclude, we observed widely distributed brain areas that primarily organize conceptual representation along taxonomic 
structures, with thematic relations further embedded in the taxonomic categories. Only in the TPJ were thematic effects as strong as the taxonomic effects, which might be related to its relevance in semantic features that are central to thematic event formation such as space and action. This shared brain system for taxonomic and thematic dimensions may reflect a unified, feature-based integration mechanism for different types of semantic relations.

\section{References}

Anderson AJ, Murphy B, Poesio M (2014) Discriminating taxonomic categories and domains in mental simulations of concepts of varying concreteness. J Cogn Neurosci 26:658-681. CrossRef Medline

Bálint R (1909) Seelenlahmungs des' Schauens', optische ataxie, raumliche storung der aufmerksamkeit. Monatsschr Psychiat Neurol 25:51-81. CrossRef

Binder JR (2016) In defense of abstract conceptual representations. Psychon Bull Rev 23:1096-1108. CrossRef Medline

Binder JR, Desai RH, Graves WW, Conant LL (2009) Where is the semantic system? A critical review and meta-analysis of 120 functional neuroimaging studies. Cereb Cortex 19:2767-2796. CrossRef Medline

Binder JR, Conant LL, Humphries CJ, Fernandino L, Simons SB, Aguilar M, Desai RH (2016) Toward a brain-based componential semantic representation. Cogn Neuropsychol 33:130-174. CrossRef Medline

Bracci S, Daniels N, Op de Beeck H (2017) Task context overrules objectand category-related representational content in the human parietal cortex. Cereb Cortex 27:310-321. CrossRef Medline

Busing F, Commandeur JJ, Heiser WJ, Bandilla W, Faulbaum F (1997) PROXSCAL: a multidimensional scaling program for individual differences scaling with constraints. Softstat 97:67-74.

Chiu YC, Esterman M, Han Y, Rosen H, Yantis S (2011) Decoding taskbased attentional modulation during face categorization. J Cogn Neurosci 23:1198-1204. CrossRef Medline

Clarke A, Tyler LK (2014) Object-specific semantic coding in human perirhinal cortex. J Neurosci 34:4766-4775. CrossRef Medline

Dale AM (1999) Optimal experimental design for event-related fMRI. Hum Brain Mapp 8:109-114. CrossRef Medline

Dalrymple KA, Barton JJ, Kingstone A (2013) A world unglued: simultanagnosia as a spatial restriction of attention. Front Hum Neurosci 7:145. CrossRef Medline

De Falco E, Ison MJ, Fried I, Quian Quiroga R (2016) Long-term coding of personal and universal associations underlying the memory web in the human brain. Nat Commun 7:13408. CrossRef Medline

Devereux BJ, Clarke A, Marouchos A, Tyler LK (2013) Representational similarity analysis reveals commonalities and differences in the semantic processing of words and objects. J Neurosci 33:18906-18916. CrossRef Medline

de Zubicaray GI, Hansen S, McMahon KL (2013) Differential processing of thematic and categorical conceptual relations in spoken word production. J Exp Psychol Gen 142:131-142. CrossRef Medline

de Zubicaray G, Johnson K, Howard D, McMahon K (2014) A perfusion fMRI investigation of thematic and categorical context effects in the spoken production of object names. Cortex 54:135-149. CrossRef Medline

Dilks DD, Julian JB, Paunov AM, Kanwisher N (2013) The occipital place area is causally and selectively involved in scene perception. J Neurosci 33:1331-1336a. CrossRef Medline

Estes Z, Golonka S, Jones LL (2011) Thematic thinking: the apprehension and consequences of thematic relations. Psychology of Learning and Motivation-Advances in Research and Theory 54:249. CrossRef

Fairhall SL, Caramazza A (2013a) Category-selective neural substrates for person-and place-related concepts. Cortex 49:2748-2757. CrossRef Medline

Fairhall SL, Caramazza A (2013b) Brain regions that represent amodal conceptual knowledge. J Neurosci 33:10552-10558. CrossRef Medline

Geng J, Schnur TT (2016) Role of features and categories in the organization of object knowledge: evidence from adaptation fMRI. Cortex 78:174-194. CrossRef Medline

Georgopoulos AP, Schwartz AB, Kettner RE (1986) Neuronal population coding of movement direction. Science 233:1416-1419. CrossRef Medline

Georgopoulos AP, Kettner RE, Schwartz AB (1988) Primate motor cortex and free arm movements to visual targets in 3D space. II. coding of the direction of movement by a neuronal population. J Neurosci 8:29282937. Medline

Goodale MA, Milner AD (1992) Separate visual pathways for perception and action. Trends Neurosci 15:20-25. CrossRef Medline

Grinband J, Wager TD, Lindquist M, Ferrera VP, Hirsch J (2008) Detection of time-varying signals in event-related fMRI designs. Neuroimage 43: 509-520. CrossRef Medline

Hjortkjær J, Kassuba T, Madsen KH, Skov M, Siebner HR (2018) Taskmodulated cortical representations of natural sound source categories. Cereb Cortex 28:295-306. CrossRef Medline

Iacoboni M, Dapretto M (2006) The mirror neuron system and the consequences of its dysfunction. Nat Rev Neurosci 7:942-951. CrossRef Medline

Jackson RL, Hoffman P, Pobric G, Lambon Ralph MA (2015) The nature and neural correlates of semantic association versus conceptual similarity. Cereb Cortex 25:4319-4333. CrossRef Medline

Kalénine S, Buxbaum LJ (2016) Thematic knowledge, artifact concepts, and the left posterior temporal lobe: where action and object semantics converge. Cortex 82:164-178. CrossRef Medline

Kalénine S, Peyrin C, Pichat C, Segebarth C, Bonthoux F, Baciu M (2009) The sensory-motor specificity of taxonomic and thematic conceptual relations: a behavioral and fMRI study. Neuroimage 44:1152-1162. CrossRef Medline

Kalénine S, Mirman D, Middleton EL, Buxbaum LJ (2012) Temporal dynamics of activation of thematic and functional knowledge during conceptual processing of manipulable artifacts. J Exp Psychol Learn Mem Cogn 38:1274-1295. CrossRef Medline

Kotz SA, Cappa SF, von Cramon DY, Friederici AD (2002) Modulation of the lexical-semantic network by auditory semantic priming: an eventrelated functional MRI study. Neuroimage 17:1761-1772. CrossRef Medline

Kriegeskorte N, Goebel R, Bandettini P (2006) Information-based functional brain mapping. Proc Natl Acad Sci U S A 103:3863-3868. CrossRef Medline

Kriegeskorte N, Mur M, Bandettini P (2008) Representational similarity analysis: connecting the branches of systems neuroscience. Front Syst Neurosci 2:4. CrossRef Medline

Kuchinke L, Meer Ev, Krueger F (2009) Differences in processing of taxonomic and sequential relations in semantic memory: an fMRI investigation. Brain Cogn 69:245-251. CrossRef Medline

Lewis GA, Poeppel D, Murphy GL (2015) The neural bases of taxonomic and thematic conceptual relations: an MEG study. Neuropsychologia 68: 176-189. CrossRef Medline

Mellet E, Tzourio N, Denis M, Mazoyer B (1995) A positron emission tomography study of visual and mental spatial exploration. J Cogn Neurosci 7:433-445. CrossRef Medline

Mirman D, Graziano KM (2012) Damage to temporo-parietal cortex decreases incidental activation of thematic relations during spoken word comprehension. Neuropsychologia 50:1990-1997. CrossRef Medline

Mirman D, Landrigan JF, Britt AE (2017) Taxonomic and thematic semantic systems. Psychol Bull 143:499-520. CrossRef Medline

Misaki M, Kim Y, Bandettini PA, Kriegeskorte N (2010) Comparison of multivariate classifiers and response normalizations for patterninformation fMRI. Neuroimage 53:103-118. CrossRef Medline

Mur M, Bandettini PA, Kriegeskorte N (2009) Revealing representational content with pattern-information fMRI-an introductory guide. Soc Cogn Affect Neurosci 4:101-109. CrossRef Medline

Murphy K, Bodurka J, Bandettini PA (2007) How long to scan? the relationship between fMRI temporal signal to noise ratio and necessary scan duration. Neuroimage 34:565-574. CrossRef Medline

Nastase SA, Connolly AC, Oosterhof NN, Halchenko YO, Guntupalli JS, Visconti di Oleggio Castello M, Gors J, Gobbini MI, Haxby JV (2017) Attention selectively reshapes the geometry of distributed semantic representation. Cereb Cortex 27:4277-4291. CrossRef Medline

Nichols TE, Holmes AP (2002) Nonparametric permutation tests for functional neuroimaging: a primer with examples. Hum Brain Mapp 15:1-25. CrossRef Medline

Oldfield RC (1971) The assessment and analysis of handedness: the edinburgh inventory. Neuropsychologia 9:97-113. CrossRef Medline

Quiroga RQ (2012) Concept cells: the building blocks of declarative memory functions. Nat Rev Neurosci 13:587-597. CrossRef Medline 
Ralph MA, Jefferies E, Patterson K, Rogers TT (2017) The neural and computational bases of semantic cognition. Nat Rev Neurosci 18:42-55. CrossRef Medline

Rogers TT, McClelland JL (2004) Semantic cognition: a parallel distributed processing approach. Cambridge, MA: MIT.

Rudrauf D, Mehta S, Bruss J, Tranel D, Damasio H, Grabowski TJ (2008) Thresholding lesion overlap difference maps: application to category-related naming and recognition deficits. Neuroimage 41:970-984. CrossRef Medline

Sachs O, Weis S, Krings T, Huber W, Kircher T (2008a) Categorical and thematic knowledge representation in the brain: neural correlates of taxonomic and thematic conceptual relations. Neuropsychologia 46:409418. CrossRef Medline

Sachs O, Weis S, Zellagui N, Huber W, Zvyagintsev M, Mathiak K, Kircher T (2008b) Automatic processing of semantic relations in fMRI: neural activation during semantic priming of taxonomic and thematic categories. Brain Res 1218:194-205. CrossRef Medline

Sachs O, Weis S, Zellagui N, Sass K, Huber W, Zvyagintsev M, Mathiak K, Kircher T (2011) How different types of conceptual relations modulate brain activation during semantic priming. J Cogn Neurosci 23:12631273. CrossRef Medline

Sass K, Sachs O, Krach S, Kircher T (2009) Taxonomic and thematic categories: neural correlates of categorization in an auditory-to-visual priming task using fMRI. Brain Res 1270:78-87. CrossRef Medline

Schwartz MF, Kimberg DY, Walker GM, Brecher A, Faseyitan OK, Dell GS, Mirman D, Coslett HB (2011) Neuroanatomical dissociation for taxonomic and thematic knowledge in the human brain. Proc Natl Acad Sci U S A 108:8520-8524. CrossRef Medline
Shinkareva SV, Malave VL, Mason RA, Mitchell TM, Just MA (2011) Commonality of neural representations of words and pictures. Neuroimage 54:2418-2425. CrossRef Medline

Simanova I, Hagoort P, Oostenveld R, van Gerven MA (2014) Modalityindependent decoding of semantic information from the human brain. Cereb Cortex 24:426-434. CrossRef Medline

Stokes M, Thompson R, Nobre AC, Duncan J (2009) Shape-specific preparatory activity mediates attention to targets in human visual cortex. Proc Natl Acad Sci U S A 106:19569-19574. CrossRef Medline

Sun H, Huang J, Sun D, Li D, Xing H (1997) Introduction to language corpus system of modern Chinese study. In: Paper collection for the fifth world Chinese teaching symposium, pp 459-466. Beijing: Peking University.

Todd MT, Nystrom LE, Cohen JD (2013) Confounds in multivariate pattern analysis: theory and rule representation case study. Neuroimage 77 : 157-165. CrossRef Medline

Van Essen DC (2005) A population-average, landmark-and surface-based (PALS) atlas of human cerebral cortex. Neuroimage 28:635-662. CrossRef Medline

Xu Y, Lin Q, Han Z, He Y, Bi Y (2016) Intrinsic functional network architecture of human semantic processing: modules and hubs. Neuroimage 132:542-555. CrossRef Medline

Xu Y, He Y, Bi Y (2017) A tri-network model of human semantic processing. Front Psychol 8:1538. CrossRef Medline

Yarkoni T, Barch DM, Gray JR, Conturo TE, Braver TS (2009) BOLD correlates of trial-by-trial RT variability in gray and white matter: a multistudy fMRI analysis. PLoS One 4:e4257. CrossRef Medline 